\title{
ELLIPTIC DIVISIBILITY SEQUENCES, SQUARES AND CUBES
}

\author{
BETÜL GEZER
}

\begin{abstract}
Elliptic divisibility sequences (EDSs) are generalizations of a class of integer divisibility sequences called Lucas sequences. There has been much interest in cases where the terms of Lucas sequences are squares or cubes. In this work, using the Tate normal form having one parameter of elliptic curves with torsion points, the general terms and periods of all elliptic divisibility sequences with a zero term are given in terms of this parameter by means of Mazur's theorem, and it is shown that which term of $h_{n}$ of an EDS can be a square or a cube by using the general terms of these sequences.
\end{abstract}

AMS Subject Classification 2010: 11B37, 11B39, 11B83, 11G05.

Keywords: Elliptic curves, torsion points, Tate normal forms, elliptic divisibility sequences, squares, cubes.

Date: 21. 09. 2011

\section{INTRODUCTION}

A divisibility sequence is a sequence $\left(h_{n}\right)(n \in \mathbb{N})$ of integers with the property that $h_{n} \mid h_{m}$ if $n \mid m$. There are also elliptic divisibility sequences satisfying a nonlinear recurrence relation that comes from the recursion formula for elliptic division polynomials associated to an elliptic curve.

An elliptic divisibility sequence (EDS) is a sequence $\left(h_{n}\right)$ of integers satisfying a nonlinear recurrence relation

$$
h_{m+n} h_{m-n}=h_{m+1} h_{m-1} h_{n}^{2}-h_{n+1} h_{n-1} h_{m}^{2}
$$

and such that $h_{n}$ divides $h_{m}$ whenever $n$ divides $m$ for all $m \geq n \geq 1$. The recurrence relation (1.1) is less straightforward than a linear recurrence.

EDSs are generalizations of a class of integer divisibility sequences called Lucas sequences. EDSs are interesting because they were the first nonlinear divisibility sequences to be studied. Morgan Ward wrote several papers detailing the arithmetic theory of EDSs [25, 26].

In order to calculate terms, there are two useful formulas (known as duplication formulas) which are obtained from (1.1) by setting first $m=n+1, n=m$ and then $m=n+1, n=m-1$ :

$$
\begin{aligned}
& h_{2 n+1}=h_{n+2} h_{n}^{3}-h_{n-1} h_{n+1}^{3}, \\
& h_{2 n} h_{2}=h_{n}\left(h_{n+2} h_{n-1}^{2}-h_{n-2} h_{n+1}^{2}\right)
\end{aligned}
$$

for all $n \in \mathbb{N}$. A solution of (1.1) is proper if $h_{0}=0, h_{1}=1$, and $h_{2} h_{3} \neq 0$. Such a proper solution will be an EDS if and only if $h_{2}, h_{3}, h_{4}$ are integers with $h_{2} \mid h_{4}$. The sequence $\left(h_{n}\right)$ with initial values $h_{0}=0, h_{1}=1, h_{2}, h_{3}$ and $h_{4}$ is denoted by $\left[1 ; h_{2} ; h_{3} ; h_{4}\right]$. 
Note that in order to compute the term $h_{n}$ of the elliptic divisibility sequence $\left(h_{n}\right)$ by any of the formulas above we need to know all previous terms before $h_{n}$, but this would be very wasteful.

Ward gave formulas for a very special case of the EDSs whose second or third term is zero which are called improper sequences. To over come this problem we give general terms of the EDSs with zero terms. This will also help us to determine the square and cube terms in these sequences as described in the following sections.

One of the well known theorems in the theory of elliptic curves is Mazur's theorem which states that there is no points of order 11 and greater than 12 on an elliptic curve over $\mathbb{Q}$. Hence, the rank of the elliptic divisibility sequences associated to elliptic curves with points of finite order can not be 11 and greater than 12. Therefore, any of second, ..., tenth and twelfth terms of an elliptic divisibility sequence can be zero.

In this work we will answer the following questions:

- What are the initial values of the EDSs with second, ..., tenth and twelfth term zero?

- Is there any formula to calculate the terms of the EDSs with second, ..., tenth and twelfth term zero which is more useful than relations above, i.e., what are the general terms of these sequences?

- What are the periods of these sequences?

- Which terms of these sequences can be a square or a cube?

The first two questions are discussed in Section 3. The initial values of the EDSs with zero terms and the general terms of these sequences are given in Theorem 3.1, and Theorem 3.2, respectively. The third question is considered in Section 4, and the periods of these sequences are given in Theorem 4.2. In Section 5, the question of when a term of an elliptic divisibility sequence with zero terms can be a square or a cube was discussed in detail.

\section{Some PRELIMINARIES ON ELLIPTIC CURVES AND EDSs}

An elliptic curve over $\mathbb{Q}$, is the set of solutions to an equation of the normal form, or generalized Weierstrass form,

$$
E: y^{2}+a_{1} x y+a_{3} y=x^{3}+a_{2} x^{2}+a_{4} x+a_{6}
$$

with coefficients $a_{1}, \ldots, a_{6}$ in $\mathbb{Q}$. The set of all solutions $(x, y) \in \mathbb{Q} \times \mathbb{Q}$ to the equation (2.1) together with the point $\mathbf{O}$, called the point at infinity, is denoted by $E(\mathbb{Q})$ and called the set of $\mathbb{Q}$-rational points on $E$. The set of $\mathbb{Q}$-points on $E$ forms an abelian subgroup of $E$ known as the Mordell-Weil group of $E$. For more details on elliptic curves in general, see 21, 22. One of the most important theorems in the theory of elliptic curves is Mordell-Weil theorem, which implies that, if $\mathbb{K}$ is a number field containing $\mathbb{Q}$, then $E(\mathbb{K})$ is a finitely generated abelian group. Also, the Mordell-Weil theorem shows that $E_{\text {tors }}(\mathbb{K})$, the torsion subgroup of $E(\mathbb{K})$, is finitely generated and abelian, hence it is finite, since its generators are of finite order. It is always interesting to characterize the torsion subgroup of a given elliptic curve. The question of a uniform bound on $E_{\text {tors }}(\mathbb{Q})$ was studied from the point of view of modular curves by Shimura, Ogg, and others. In 1976, B. Mazur proved the following strongest result which had been conjectured by Ogg:

Theorem 2.1. (Mazur [12]) Let $E$ be an elliptic curve defined over $\mathbb{Q}$. Then the torsion subgroup $E_{\text {tors }}(\mathbb{Q})$ is either isomorphic to $\mathbb{Z} / N \mathbb{Z}$ for $N=1,2, \ldots, 10,12$ or 
to $\mathbb{Z} / 2 \mathbb{Z} \times \mathbb{Z} / 2 N \mathbb{Z}$ for $N=1,2,3,4$. Further, each of these groups does occur as an $E_{\text {tors }}(\mathbb{Q})$.

One of the aim of this work is to give the general terms of the elliptic divisibility sequences with this property by using Tate normal form with one parameter of an elliptic curve according to this parameter.

The Tate normal form of an elliptic curve $E$ with point $P=(0,0)$ is defined by

$$
E: y^{2}+(1-c) x y-b y=x^{3}-b x^{2} .
$$

If an elliptic curve in normal form has a point of order $N>3$, then admissible change of variables transforms the curve to the Tate normal form, in this case the point $P=(0,0)$ is a torsion point of maximal order. Especially, if we want a classification with respect to the order of the torsion points, the use of Tate normal form of elliptic curves is unavoidable.

In [11, Kubert listed that there is one parameter family of elliptic curves $E$ defined over $\mathbb{Q}$ with a torsion point of order $N$ where $N=4, \ldots, 10,12$. Most cases are proved by Husemöller, 10. Also some algorithms are given by using the existence of such a family, [6]. To decide when an elliptic curve defined over $\mathbb{Q}$ has a point of given order $N$, we need a result on parametrization of torsion structures:

Theorem 2.2. 6] Every elliptic curve with a point $P$ of order $N=4, \ldots, 10,12$ can be written in the following Tate normal form

$$
E: y^{2}+(1-c) x y-b y=x^{3}-b x^{2},
$$

with the following relations:

1. If $N=4, b=\alpha, c=0$.

2. If $N=5, b=\alpha, c=\alpha$.

3. If $N=6, b=\alpha+\alpha^{2}, c=\alpha$.

4. If $N=7, b=\alpha^{3}-\alpha^{2}, c=\alpha^{2}-\alpha$.

5. If $N=8, b=(2 \alpha-1)(\alpha-1), c=b / \alpha$.

6. If $N=9, c=\alpha^{2}(\alpha-1), b=c(\alpha(\alpha-1)+1)$.

7. If $N=10, c=\left(2 \alpha^{3}-3 \alpha^{2}+\alpha\right) /\left(\alpha-(\alpha-1)^{2}\right), b=c \alpha^{2} /\left(\alpha-(\alpha-1)^{2}\right)$.

8. If $N=12, c=\left(3 \alpha^{2}-3 \alpha+1\right)\left(\alpha-2 \alpha^{2}\right) /(\alpha-1)^{3}, b=c\left(-2 \alpha^{2}+2 \alpha-1\right) /(\alpha-1)$.

Theorem 2.2 states that, if any elliptic curve has a point of finite order then this curve is birationally equivalent to one of the Tate normal forms given in the theorem above. Therefore, in this work, we are only interested in the elliptic curves in Tate normal forms with one integer parameter $\alpha$ and general terms of the elliptic divisibility sequences are given depending on this integer parameter $\alpha$.

Ward proved, in 26, that birationally equivalent elliptic curves are associated to equivalent elliptic divisibility sequences, so it is not a restriction to give the general terms by using Tate normal forms, that is, we will be giving general terms of all elliptic divisibility sequences with zero terms under this equivalence.

The relation between an elliptic curve and an elliptic divisibility sequence is given by Morgan Ward, see for details and formulas, [26]. Ward proved that elliptic divisibility sequences arise as values of the division polynomials of an elliptic curve, i.e., if $P=(x, y)$ is a rational point on an elliptic curve $E$ over $\mathbb{Q}$ then the elliptic divisibility sequence $\left(h_{n}\right)$ is defined by $h_{n}=\psi_{n}(x, y)$ for $n \in \mathbb{N}$ where $\psi_{n}$ is the $n$-th division polynomial of $E$. Therefore, if $E$ is an elliptic curve over $\mathbb{Q}$ then the initial values of the elliptic divisibility sequence are given by the coefficients of an elliptic curve. Conversely, if $\left(h_{n}\right)$ is an elliptic divisibility sequence in which neither $h_{2}$ nor 
$h_{3}$ is zero then there exists an elliptic curve $E$ and the coefficients of the elliptic curve are given by the initial values of the sequence. In this paper, under this fact, we first give initial values and the general terms of an elliptic divisibility sequence associated to an elliptic curve in Tate normal form with a torsion point $P$. We will now give a short account of material about elliptic divisibility sequences, for more detailed information about these sequences in general, see [4, 5, 20, 24, 25, 26.

Two elliptic divisibility sequences $\left(h_{n}\right)$ and $\left(h_{n}^{\prime}\right)$ are said to be equivalent if there exists a rational $\omega$ such that

$$
h_{n}^{\prime}=\omega^{n^{2}-1} h_{n}
$$

for all $n \in \mathbb{N}$.

Since we are interested in sequences with zero terms, i.e., the sequences in certain ranks, we have to know the concept of the rank of an EDS:

An integer $m$ is said to be a divisor of the sequence $\left(h_{n}\right)$ if it divides some term $h_{k}$ with $k>0$. Let $m$ be a divisor of $\left(h_{n}\right)$. If $\rho$ is an integer such that $m \mid h_{\rho}$ and there is no integer $j$ such that $j$ is a divisor of $\rho$ with $m \mid h_{j}$, then $\rho$ is said to be the rank of apparition of $m$ in $\left(h_{n}\right)$. Ward established that the multiples of $\rho$ are regularly spaced in $\left(h_{n}\right)$ in the following theorem.

Theorem 2.3. [26] Let $p$ be a prime divisor of an elliptic divisibility sequence $\left(h_{n}\right)$, and let $\rho$ be its smallest rank of apparition. Let $h_{\rho+1} \not \equiv 0(p)$. Then

$$
h_{n} \equiv 0(p) \text { if and only if } n \equiv 0(\rho) .
$$

The following theorem shows us that the initial values of the EDS given by the coefficients of the elliptic curve.

Theorem 2.4. 20] Let $\left(h_{n}\right)$ be an elliptic divisibility sequence. Then the elliptic curves $E: y^{2}+a_{1} x y+a_{3} y=x^{3}+a_{2} x^{2}+a_{4} x$ where $a_{1}, a_{2}, a_{3}, a_{4} \in \mathbb{Q}$, associated to $\left(h_{n}\right)$ are precisely those with:

$$
\begin{aligned}
& h_{2}=a_{3}, \\
& h_{3}=a_{2} a_{3}^{2}-a_{4}^{2}-a_{1} a_{3} a_{4} \\
& h_{4}=2 a_{3} a_{4} h_{3}+a_{1} a_{3}^{2} h_{3}-a_{3}^{5} .
\end{aligned}
$$

\section{The initial VAlues and the general terms of the EDSs}

The problem of finding the general terms of the elliptic divisibility sequences whose second (resp. third, fourth, fifth, sixth) term is zero are given in 8 . However, it was seen that the general terms of the other sequences with zero terms can not be easily obtained.

In this paper we first give the general terms of all elliptic divisibility sequences with zero terms by using Tate normal form of an elliptic curve which has a torsion point $P=(0,0)$. According to the Mazur's theorem, we know that if $\left(h_{n}\right)$ is an elliptic divisibility sequence, in which $h_{N}=0$ for some minimal index $N$ then $N \in$ $\{2, \ldots, 10,12\}$. The aim of this section is to give general terms of these sequences. Naturally, it is sufficient to give the general terms only for the sequences with one of the terms $h_{2}, \ldots, h_{10}, h_{12}$ are zero. But we begin by the case $N>3$, since we use Tate normal form of an elliptic curve. The general terms of improper elliptic divisibility sequences where $h_{2}$ or $h_{3}$ is equal to zero will be discussed at the end of this section and general terms of them will be given in Theorem 3.3. In the 
following theorem, by using the Tate normal form of an elliptic curve, the initial values of the sequences with zero terms are given for $N>3$.

Theorem 3.1. Let $\left(h_{n}\right)$ be an elliptic divisibility sequence in which $h_{N}=0$ for some minimal index $N \in\{4, \ldots, 10,12\}$. Then the initial values of $\left(h_{n}\right)$ with integer parameter $\alpha$ given by the following:

1. If $N=4,\left[1 ;-\alpha ;-\alpha^{3} ; 0\right]$.

2. If $N=5,\left[1 ;-\alpha ;-\alpha^{3} ; \alpha^{6}\right]$.

3. If $N=6,\left[1 ;-\alpha(\alpha+1) ;-\alpha^{3}(\alpha+1)^{3} ; \alpha^{6}(\alpha+1)^{5}\right]$.

4. If $N=7,\left[1 ;-\alpha^{2}(\alpha-1) ;-\alpha^{6}(\alpha-1)^{3} ; \alpha^{11}(\alpha-1)^{6}\right]$.

5. If $N=8,\left[1 ;-\alpha^{3} \beta ;-\alpha^{8} \beta^{3} ; \alpha^{14} \beta^{6}\right]$, where $\xi=(\alpha-1)(2 \alpha-1)$.

6. If $N=9,\left[1 ;-\alpha^{2}(\alpha-1) \gamma ;-\alpha^{6}(\alpha-1)^{3} \gamma^{3} ; \alpha^{12}(\alpha-1)^{6} \gamma^{5}\right]$, where $\gamma=\alpha^{2}-\alpha+1$.

7. If $N=10,\left[1 ;-\alpha^{3} \delta \zeta^{4} ;-\alpha^{9} \delta^{3} \zeta^{10} ; \quad \alpha^{16} \delta^{6} \zeta^{19}\right]$, where $\zeta=\alpha-(\alpha-1)^{2}$ and $\delta=(\alpha-1)(2 \alpha-1)$.

8. If $N=12,\left[1 ;-(\alpha-1)^{8} \lambda \theta ;-(\alpha-1)^{20} \lambda^{3} \theta^{3} ;(\alpha-1)^{37} \lambda^{6} \theta^{5}\right]$, where $\lambda=\left(3 \alpha^{2}-\right.$ $3 \alpha+1)\left(\alpha-2 \alpha^{2}\right)$ and $\theta=2 \alpha-2 \alpha^{2}-1$.

Proof. 1. We first consider an elliptic curve $E$ with a point $P$ of order $N=4$. Then by Theorem 2.2, the Tate normal form of $E$ is

$$
E: y^{2}+x y-\alpha y=x^{3}-\alpha x^{2} .
$$

By Theorem 2.4, $E$ is associated to the elliptic sequence $\left(h_{n}\right)$ and the initial values of the sequence are

$$
h_{1}=1, h_{2}=-\alpha, h_{3}=-\alpha^{3}, h_{4}=0 .
$$

It is known that, if $P$ is an integer point, and the coefficients $a_{i}$ of the elliptic curve are integers, then the values $h_{n}$ are integers, and have the divisibility property, that is, $\left(h_{n}\right)$ is an EDS. Therefore coefficients $\alpha$ in (3.1) must be an integer, since we want to work with elliptic divisibility sequences.

2. Similarly for $N=5$, we have

$$
E: y^{2}+(1-\alpha) x y-\alpha y=x^{3}-\alpha x^{2},
$$

and so, the initial values of the sequence are

$$
h_{1}=1, h_{2}=-\alpha, h_{3}=-\alpha^{3}, h_{4}=\alpha^{6} .
$$

3. For $N=6$, we have

$$
E: y^{2}+(1-\alpha) x y-\alpha(\alpha+1) y=x^{3}-\alpha(\alpha+1) x^{2},
$$

and so, the initial values of the sequence are

$$
h_{1}=1, h_{2}=-\alpha(\alpha+1), h_{3}=-\alpha^{3}(\alpha+1)^{3}, h_{4}=\alpha^{6}(\alpha+1)^{5} .
$$

4. For $N=7$, we have

$$
E: y^{2}+\left(1-\alpha^{2}+\alpha\right) x y-\left(\alpha^{3}-\alpha^{2}\right) y=x^{3}-\left(\alpha^{3}-\alpha^{2}\right) x^{2},
$$

and so, the initial values of the sequence are

$$
h_{1}=1, h_{2}=-\alpha^{2}(\alpha-1), h_{3}=-\alpha^{6}(\alpha-1)^{3}, h_{4}=\alpha^{11}(\alpha-1)^{6} .
$$

5. Now let $E$ be an elliptic curve in normal form with a point $P$ of order $N=8$. Then by Theorem 2.2, the Tate normal form of $E$ is

$$
E: y^{2}+(1-(2 \alpha-1)(\alpha-1) / \alpha) x y-(2 \alpha-1)(\alpha-1) y=x^{3}-(2 \alpha-1)(\alpha-1) x^{2} \text {. }
$$


Since we work with elliptic divisibility sequences, coefficients of the elliptic curve must be integer, so we transform the elliptic curve $E$ to a birationally equivalent curve $\widetilde{E}$ under admissible change of variables

$$
\widetilde{E}: y^{2}+(\alpha-\xi) x y-\alpha^{3} \xi y=x^{3}-\alpha^{2} \xi x^{2}
$$

where $\xi=(2 \alpha-1)(\alpha-1)$. By Theorem 2.4, $\widetilde{E}$ is associated to the elliptic divisibility sequence $\left(h_{n}\right)$ and the initial values of the sequence are

$h_{1}=1, h_{2}=-\alpha^{3}(\alpha-1)(2 \alpha-1), h_{3}=-\alpha^{8}(\alpha-1)^{3}(2 \alpha-1)^{3}, h_{4}=\alpha^{14}(\alpha-1)^{6}(2 \alpha-1)^{6}$.

6. For $N=9$ we have

$$
E: y^{2}+(1-c) x y-b y=x^{3}-b x^{2}
$$

where $c=\alpha^{2}(\alpha-1), b=c(\alpha(\alpha-1)+1)$, and so, the initial values of this sequence are

$$
h_{1}=1, h_{2}=-\alpha^{2}(\alpha-1) \gamma, h_{3}=-\alpha^{6}(\alpha-1)^{3} \gamma^{3}, h_{4}=\alpha^{12}(\alpha-1)^{6} \gamma^{5}
$$

where $\gamma=\alpha^{2}-\alpha+1$.

7. Now let $E$ be an elliptic curve in normal form with a point $P$ of order $N=10$. By Theorem 2.2, the Tate normal form of $E$ is

$$
E: y^{2}+(1-c) x y-b y=x^{3}-b x^{2}
$$

where $c=\alpha\left(2 \alpha^{2}-3 \alpha+1\right) /\left(\alpha-(\alpha-1)^{2}\right), b=c \alpha^{2} /\left(\alpha-(\alpha-1)^{2}\right)$ and $E$ birationally equivalent to the curve $\widetilde{E}$ under admissible change of variables given by

$$
\widetilde{E}: y^{2}+\left(\delta^{2}-\delta \zeta\right) x y-\alpha^{2} \zeta \delta^{4} y=x^{3}-\zeta \alpha^{2} x^{2},
$$

where $\zeta=(2 \alpha-1)(\alpha-1), \delta=\alpha-(\alpha-1)^{2}$. By Theorem 2.4, $\widetilde{E}$ is associated to the elliptic divisibility sequence $\left(h_{n}\right)$ and the initial values of this sequence are

$$
h_{1}=1, h_{2}=-\alpha^{3} \zeta \delta^{4}, h_{3}=-\alpha^{9} \zeta^{3} \delta^{10}, h_{4}=\alpha^{16} \zeta^{6} \delta^{19} .
$$

8. Now let $E$ be an elliptic curve in normal form with a point $P$ of order $N=12$. By Theorem 2.2, the Tate normal form of $E$ is

$$
E: y^{2}+(1-c) x y-b y=x^{3}-b x^{2},
$$

where $c=\left(3 \alpha^{2}-3 \alpha+1\right) \alpha(1-2 \alpha) /(\alpha-1)^{3}, b=c\left(2 \alpha-2 \alpha^{2}-1\right) /(\alpha-1)$ and $E$ birationally equivalent to the curve $\widetilde{E}$ under admissible change of variables given by

$$
\widetilde{E}: y^{2}+(\alpha-1)\left((\alpha-1)^{3}-\lambda\right) x y-(\alpha-1)^{8} \lambda \theta y=x^{3}-(\alpha-1)^{4} \lambda \theta x^{2},
$$

where $\lambda=\left(3 \alpha^{2}-3 \alpha+1\right)\left(\alpha-2 \alpha^{2}\right), \theta=2 \alpha-2 \alpha^{2}-1$. By Theorem 2.4, $\widetilde{E}$ is associated to the elliptic divisibility sequence $\left(h_{n}\right)$ and the initial values of this sequence are

$$
h_{1}=1, h_{2}=-(\alpha-1)^{8} \lambda \theta, h_{3}=-(\alpha-1)^{20} \lambda^{3} \theta^{3}, h_{4}=(\alpha-1)^{37} \lambda^{6} \theta^{5} .
$$

Thus we know the initial values of the sequences $\left(h_{n}\right)$ with zero terms. We now give the general terms of the sequences $\left(h_{n}\right)$ with zero terms depending on only one integer parameter $\alpha$ in the following theorem. 
Theorem 3.2. Let $\left(h_{n}\right)$ be an elliptic divisibility sequence with $N$-th term zero, i.e., with rank $N \in\{4, \ldots, 10,12\}$. Let $\xi, \gamma, \delta, \zeta, \lambda, \theta$ as in Theorem 3.1. Then the general term of $\left(h_{n}\right)$ given by the following formulas:

1. If $N=4$,

$$
h_{n}=\varepsilon \alpha^{\left\{\left(3 n^{2}-p\right) / 8\right\}}
$$

where $\varepsilon=\left\{\begin{array}{ll}+1 & \text { if } n \equiv 1,5,6(8) \\ -1 & \text { if } n \equiv 2,3,7(8),\end{array} \quad p= \begin{cases}3 & \text { if } n \equiv 1,3(4) \\ 4 & \text { if } n \equiv 2(4) .\end{cases}\right.$

2. If $N=5$,

$$
h_{n}=\varepsilon \alpha^{\left\{\left(2 n^{2}-p\right) / 5\right\}}
$$

where $\varepsilon=\left\{\begin{array}{ll}+1 & \text { if } n \equiv 1,4,7,8(10) \\ -1 & \text { if } n \equiv 2,3,6,9(10),\end{array} \quad p= \begin{cases}2 & \text { if } n \equiv 1,4(5) \\ 3 & \text { if } n \equiv 2,3(5) \text {. }\end{cases}\right.$

3. If $N=6$,

$$
h_{n}=\varepsilon \alpha^{\left\{\left(5 n^{2}-p\right) / 12\right\}}(\alpha+1)^{\left\{\left(n^{2}-k\right) / 3\right\}}
$$

where $\varepsilon=\left\{\begin{array}{ll}+1 & \text { if } n \equiv 1,4,5,9,10(12) \\ -1 & \text { if } n \equiv 2,3,7,8,11(12),\end{array} \quad\right.$ and

$$
p=\left\{\begin{array}{ll}
5 & \text { if } n \equiv 1,5(6) \\
8 & \text { if } n \equiv 2,4(6) \\
9 & \text { if } n \equiv 3(6),
\end{array} \quad k= \begin{cases}1 & \text { if } n \equiv 1,2,4,5(6) \\
0 & \text { if } n \equiv 3(6) .\end{cases}\right.
$$

4. If $N=7$,

$$
h_{n}=\varepsilon \alpha^{\left\{\left(5 n^{2}-p\right) / 7\right\}}(\alpha-1)^{\left\{\left(3 n^{2}-q\right) / 7\right\}}
$$

where $\varepsilon=\left\{\begin{array}{ll}+1 & \text { if } n \equiv 1,4,5(7) \\ -1 & \text { if } n \equiv 2,3,6(7),\end{array} \quad\right.$ and

$$
p=\left\{\begin{array}{ll}
5 & \text { if } n \equiv 1,6(7) \\
6 & \text { if } n \equiv 2,5(7) \\
3 & \text { if } n \equiv 3,4(7),
\end{array} \quad q= \begin{cases}3 & \text { if } n \equiv 1,6(7) \\
5 & \text { if } n \equiv 2,5(7) \\
6 & \text { if } n \equiv 3,4(7)\end{cases}\right.
$$

5. If $N=8$,

$$
h_{n}=\varepsilon \alpha^{\left\{\left(15 n^{2}-p\right) / 16\right\}}(\alpha-1)^{\left\{\left(7 n^{2}-q\right) / 16\right\}}(2 \alpha-1)^{\left\{\left(3 n^{2}-k\right) / 8\right\}}
$$

where $\varepsilon=\left\{\begin{array}{ll}+1 & \text { if } n \equiv 1,4,5,9,10,13,14(16) \\ -1 & \text { if } n \equiv 2,3,6,7,11,12,15(16),\end{array} \quad\right.$ and

$p=\left\{\begin{array}{ll}15 & \text { if } n \equiv 1,7(8) \\ 12 & \text { if } n \equiv 2,6(8) \\ 7 & \text { if } n \equiv 3,5(8) \\ 16 & \text { if } n \equiv 4(8),\end{array} \quad q=\left\{\begin{array}{ll}7 & \text { if } n \equiv 1,7(8) \\ 12 & \text { if } n \equiv 2,6(8) \\ 15 & \text { if } n \equiv 3,5(8) \\ 16 & \text { if } n \equiv 4(8),\end{array} \quad k= \begin{cases}3 & \text { if } n \equiv 1,3,5,7(8) \\ 4 & \text { if } n \equiv 2,6(8) \\ 0 & \text { if } n \equiv 4(8) .\end{cases}\right.\right.$

6. If $N=9$,

$$
h_{n}=\varepsilon \alpha^{\left\{\left(7 n^{2}-p\right) / 9\right\}}(\alpha-1)^{\left\{\left(4 n^{2}-q\right) / 9\right\}} \gamma^{\left\{\left(n^{2}-k\right) / 3\right\}}
$$


where $\varepsilon=\left\{\begin{array}{ll}+1 & \text { if } n \equiv 1,4,5,8,11,12,15,16(18) \\ -1 & \text { if } n \equiv 2,3,6,7,10,13,14,17(18),\end{array} \quad\right.$ and

$p=\left\{\begin{array}{ll}7 & \text { if } n \equiv 1,8(9) \\ 10 & \text { if } n \equiv 2,7(9) \\ 9 & \text { if } n \equiv 3,6(9) \\ 4 & \text { if } n \equiv 4,5(9)\end{array} \quad q=\left\{\begin{array}{ll}4 & \text { if } n \equiv 1,8(9) \\ 7 & \text { if } n \equiv 2,7(9) \\ 9 & \text { if } n \equiv 3,6(9) \\ 10 & \text { if } n \equiv 4,5(9)\end{array} \quad k= \begin{cases}0 & \text { if } n \equiv 3,6(9) \\ 1 & \text { otherwise. }\end{cases}\right.\right.$

7. If $N=10$,

$$
h_{n}=\varepsilon \alpha^{\left\{\left(21 n^{2}-p\right) / 20\right\}}(\alpha-1)^{\left\{\left(9 n^{2}-q\right) / 20\right\}}(2 \alpha-1)^{\left\{\left(2 n^{2}-k\right) / 5\right\}} \delta^{\left\{\left(5 n^{2}-s\right) / 4\right\}}
$$

where $\varepsilon=\left\{\begin{array}{ll}+1 & \text { if } n \equiv 1,4,5,8,9,13,14,17,18(20) \\ -1 & \text { if } n \equiv 2,3,6,7,11,12,15,16,19(20),\end{array} \quad\right.$ and

$$
\begin{aligned}
& p=\left\{\begin{array}{ll}
21 & \text { if } n \equiv 1,9(10) \\
24 & \text { if } n \equiv 2,8(10) \\
9 & \text { if } n \equiv 3,7(10) \\
16 & \text { if } n \equiv 4,6(10) \\
25 & \text { if } n \equiv 5(10),
\end{array} \quad q= \begin{cases}9 & \text { if } n \equiv 1,9(10) \\
16 & \text { if } n \equiv 2,8(10) \\
21 & \text { if } n \equiv 3,7(10) \\
24 & \text { if } n \equiv 4,6(10) \\
25 & \text { if } n \equiv 5(10),\end{cases} \right. \\
& k=\left\{\begin{array}{ll}
2 & \text { if } n \equiv 1,4,6,9(10) \\
3 & \text { if } n \equiv 2,3,7,8(10) \\
0 & \text { if } n \equiv 5(10),
\end{array} \quad s= \begin{cases}5 & \text { if } n \equiv 1,3,5,7,9(10) \\
4 & \text { if } n \equiv 2,4,6,8(10) .\end{cases} \right.
\end{aligned}
$$

8. If $N=12$,

(3.9)

$$
\begin{aligned}
& h_{n}=\varepsilon \alpha^{\left\{\left(n^{2}-p\right) / 12\right\}}(\alpha-1)^{\left\{\left(59 n^{2}-q\right) / 24\right\}}(2 \alpha-1)^{\left\{\left(n^{2}-k\right) / 24\right\}} \lambda^{\left\{\left(3 n^{2}-s\right) / 8\right\}} \theta^{\left\{\left(n^{2}-t\right) / 3\right\}} \\
& \text { where } \varepsilon=\begin{array}{l}
+1 \quad \text { if } n \equiv 1,5,9,13,14,16,17,18,20,21,22(24) \\
-1 \quad \text { if } n \equiv 2,3,4,6,7,8,10,11,15,19,23(24),
\end{array} \\
& p=\left\{\begin{array} { l l } 
{ 1 } & { \text { if } n \equiv 1 , 1 1 ( 1 2 ) } \\
{ 4 } & { \text { if } n \equiv 2 , 1 0 ( 1 2 ) } \\
{ 9 } & { \text { if } n \equiv 3 , 9 ( 1 2 ) } \\
{ 1 6 } & { \text { if } n \equiv 4 , 8 ( 1 2 ) } \\
{ 1 3 } & { \text { if } n \equiv 5 , 7 ( 1 2 ) } \\
{ 1 2 } & { \text { if } n \equiv 6 ( 1 2 ) , }
\end{array} \quad \left\{\begin{array}{ll}
59 \quad \text { if } n \equiv 1,11(12) \\
44 & \text { if } n \equiv 2,10(12) \\
51 & \text { if } n \equiv 3,9(12) \\
56 & \text { if } n \equiv 4,8(12) \\
35 & \text { if } n \equiv 5,7(12) \\
60 & \text { if } n \equiv 6(12),
\end{array} \quad k= \begin{cases}1 & \text { if } n \equiv 1,5,7,11(12) \\
4 & \text { if } n \equiv 2,10(12) \\
9 & \text { if } n \equiv 3,9(12) \\
16 & \text { if } n \equiv 4,8(12) \\
12 & \text { if } n \equiv 6(12),\end{cases} \right.\right. \\
& s=\left\{\begin{array}{ll}
3 & \text { if } n \equiv 1,3,5,7,9,11(12) \\
4 & \text { if } n \equiv 2,6,10(12) \\
0 & \text { if } n \equiv 4,8(12),
\end{array} \quad t= \begin{cases}1 & \text { if } n \equiv 1,2,4,5,7,8,10,11(12) \\
0 & \text { if } n \equiv 3,6,9(12) .\end{cases} \right.
\end{aligned}
$$

Proof. 1. It is clear that the result is true for $n=5$. Hence we assume that $n>5$. If $\left(h_{n}\right)$ is an EDS, then we know that

$$
h_{n+2} h_{n-2}=h_{n+1} h_{n-1} h_{2}^{2}-h_{3} h_{1} h_{n}^{2} .
$$

We argue by induction on $n$. First suppose that $n \equiv 1(4)$ and (3.2) is true for $n+1$. Then we have

$$
h_{n+2}=-\alpha^{6 m^{2}+9 m+3}
$$


by (3.2). On the other hand we see that

$$
\begin{aligned}
h_{n-2} & =-\alpha^{6 m^{2}-3 m}, \\
h_{n} & =\alpha^{6 m^{2}+3 m}, \\
h_{n-1} & =0 .
\end{aligned}
$$

Substituting these expressions into (3.10) gives $h_{n+2}=-\alpha_{2}^{6 m^{2}+9 m+3}$. Thus we proved the equation (3.2) is true for $n+2$ which completes the proof for $n \equiv 1$ (4). Other cases can be proved in the same way.

2. It is clear that the result is true for $n=6$. Hence we assume that $n>6$. If $\left(h_{n}\right)$ is an EDS, then we know that

$$
h_{n+2} h_{n-2}=h_{n+1} h_{n-1} h_{2}^{2}-h_{3} h_{1} h_{n}^{2} .
$$

We again argue by induction using (3.3). First suppose that $n \equiv 1(5)$ and (3.3) is true for $n+1$. Then we have

$$
h_{n+2}= \begin{cases}-\alpha^{10 m^{2}+12 m+3} & \text { if } m \equiv 2,4(5) \\ \alpha^{10 m^{2}+12 m+3} & \text { if } m \equiv 1,3(5)\end{cases}
$$

by (3.3). On the other hand we see that

$$
\begin{aligned}
h_{n-2} & = \begin{cases}-\alpha^{10 m^{2}-4 m} & \text { if } m \equiv 2,4(5) \\
\alpha^{10 m^{2}-4 m} & \text { if } m \equiv 1,3(5)\end{cases} \\
h_{n} & = \begin{cases}\alpha^{10 m^{2}+4 m} & \text { if } m \equiv 2,4(5) \\
-\alpha^{10 m^{2}+4 m} & \text { if } m \equiv 1,3(5)\end{cases} \\
h_{n-1} & =0 .
\end{aligned}
$$

Substituting these expressions into (3.11) gives $h_{n+2} h_{n-2}=\alpha^{3} h_{n}^{2}$, hence we have

$$
h_{n+2}= \begin{cases}-\alpha^{10 m^{2}+12 m+3} & \text { if } m \equiv 2,4(5) \\ \alpha^{10 m^{2}+12 m+3} & \text { if } m \equiv 1,3(5) .\end{cases}
$$

Thus we proved that the equation (3.3) is true for $n+2$. Other cases can be proved in the same way.

The same proof works for the remaining parts of the theorem.

For example, in the following table, it is seen that the values $p, q, k, s$ and $t$ of the general term of the elliptic divisibility sequences with rank twelve:

According to this table, we have the following terms for $n \equiv 6$ (12):

$$
\begin{aligned}
h_{6} & =\alpha^{2}(\alpha-1)^{86}(2 \alpha-1)^{2} \lambda^{13} \delta^{12} \\
h_{18} & =-\alpha^{26}(\alpha-1)^{794}(2 \alpha-1)^{14} \lambda^{121} \delta^{108} \\
h_{30} & =\alpha^{74}(\alpha-1)^{2210}(2 \alpha-1)^{38} \lambda^{337} \delta^{300} \\
h_{42} & =-\alpha^{146}(\alpha-1)^{4334}(2 \alpha-1)^{74} \lambda^{661} \delta^{588}
\end{aligned}
$$




\begin{tabular}{|c|c|c|c|c|c|}
\hline & $p$ & $q$ & $k$ & $s$ & $t$ \\
\hline \hline$n \equiv 1(12)$ & 1 & 59 & 1 & 3 & 1 \\
\hline$n \equiv 2(12)$ & 4 & 44 & 4 & 4 & 1 \\
\hline$n \equiv 3(12)$ & 9 & 51 & 9 & 3 & 0 \\
\hline$n \equiv 4(12)$ & 16 & 56 & 16 & 0 & 1 \\
\hline$n \equiv 5(12)$ & 13 & 35 & 1 & 3 & 1 \\
\hline$n \equiv 6(12)$ & $\mathbf{1 2}$ & $\mathbf{6 0}$ & $\mathbf{- 1 2}$ & $\mathbf{4}$ & $\mathbf{0}$ \\
\hline$n \equiv 7(12)$ & 13 & 35 & 1 & 3 & 1 \\
\hline$n \equiv 8(12)$ & 16 & 56 & 16 & 0 & 1 \\
\hline$n \equiv 9(12)$ & 9 & 51 & 9 & 3 & 0 \\
\hline$n \equiv 10(12)$ & 4 & 44 & 4 & 4 & 1 \\
\hline$n \equiv 11(12)$ & 1 & 59 & 1 & 3 & 1 \\
\hline
\end{tabular}

The values $p, q, k, s$ and $t$ of the general term of the EDSs with rank twelve

In addition, if we take $\alpha=3$, the first eight terms of the EDS with rank twelve are

$$
\begin{aligned}
& 1 ;-948480 ;-53329136320512000 ;-27346122891266847865307136000000 ; \\
& 17500141386070121786711926566237801283584 ;-3319445579395304657 \\
& 0787963710047756557989077368249771884544000000000000000 ;-45938 \\
& 2422798666425039100328482290559063213427355998727370793872053 \\
& 3432969818275840000000000000000000 ;-1884349228191035614337748 \\
& 21043991142309132528994842811514985817859497226098776607161543 \\
& 9393817547813525913600000000000000000000000000 ; \ldots
\end{aligned}
$$

Remark 3.1. There are also elliptic curves with a torsion point which are not in the Tate normal form as in Theorem 2.2. For example the point $P=(0,0)$ on the elliptic curve

$$
E: y^{2}+17 x y-120 y=x^{3}-60 x^{2}
$$

is a torsion point of order eight. The initial values of elliptic divisibility sequence $\left(h_{n}\right)$ associated to the curve $E$ are

$$
h_{1}=1, h_{2}=-120, h_{3}=-864000, h_{4}=-186624000000
$$

and $h_{8}=0$, that is, the sequence has rank eight. $E$ is birationally equivalent to the curve $\widetilde{E}$ under the transformation $\left(\begin{array}{l}x \\ y\end{array}\right) \rightarrow\left(\begin{array}{l}4 x \\ 8 y\end{array}\right)$ given by

$$
\widetilde{E}: y^{2}+\frac{17}{2} x y-15 y=x^{3}-15 x^{2}
$$

which is in Tate normal form. This curve gives us an elliptic sequence, so we need to make another transformation to have an elliptic divisibility sequence. Hence we have

$$
E^{\prime}: y^{2}+34 x y-960 y=x^{3}-240 x^{2}
$$

and the initial values of elliptic divisibility sequence $\left(h_{n}^{\prime}\right)$ associated to the elliptic curve $E^{\prime}$ are

$$
h_{1}^{\prime}=1, h_{2}^{\prime}=-960, h_{3}^{\prime}=-221184000, h_{4}^{\prime}=-6115295232000000 .
$$


It can easily be seen that $\left(h_{n}\right)$ and $\left(h_{n}^{\prime}\right)$ are equivalent by taking $\omega=-2$ in the equation (2.2). So, the general terms of the elliptic divisibility sequences associated to the elliptic curves in Tate normal form are the general terms of all elliptic divisibility sequences with zero terms under the equivalence.

Remark 3.2. There is no Tate normal form of an elliptic curve with the torsion point of order two or three, but Kubert in [11, listed the elliptic curves with torsion point of order two or three are

$$
E: y^{2}=x^{3}+a x^{2}+b x
$$

and

$$
E: y^{2}+a_{1} x y+a_{3} y=x^{3}
$$

respectively. In this case, the elliptic divisibility sequences associated to an elliptic curve with the torsion point of order two or three give improper sequences and the initial values of these sequences are

$$
h_{1}=1, h_{2}=0, h_{3}=-b^{2}, h_{4}=0
$$

and

$$
h_{1}=1, h_{2}=a_{3}, h_{3}=0, h_{4}=-a_{3}^{5},
$$

respectively.

Under these considerations, an easy computation gives the general terms of the improper divisibility sequences.

Theorem 3.3. $\boldsymbol{i}$. Let $\left(h_{n}\right)$ be an elliptic divisibility sequence $\left[1 ; 0 ;-b^{2} ; 0\right]$. Then the general term of $\left(h_{n}\right)$ given by the following formula:

$$
h_{n}=\varepsilon b^{\left\{\left(n^{2}-1\right) / 4\right\}}
$$

where

$$
\varepsilon= \begin{cases}+1 & \text { if } n \equiv 1,5(8) \\ -1 & \text { if } n \equiv 3,7(8) .\end{cases}
$$

ii. Let $\left(h_{n}\right)$ be an elliptic divisibility sequence $\left[1 ; a_{3} ; 0 ;-a_{3}^{5}\right]$. Then the general term of $\left(h_{n}\right)$ given by the following formula:

$$
h_{n}=\varepsilon a_{3}^{\left\{\left(n^{2}-1\right) / 3\right\}}
$$

where

$$
\varepsilon= \begin{cases}+1 & \text { if } n \equiv 1,2(6) \\ -1 & \text { if } n \equiv 4,5(6) .\end{cases}
$$

\section{THE PERIODS OF THE EDSS}

In this section we will give the periods of all elliptic divisibility sequences with zero terms by using general terms of these sequences which are given in previous section.

A sequence $\left(s_{n}\right)$ of rational integers is said to be numerically periodic modulo $\mathrm{m}$ if there exists a positive integer $\pi$ such that

$$
s_{n+\pi} \equiv s_{n}(m)
$$

for all sufficiently large $n$. If (4.1) holds for all $n$, then $\left(s_{n}\right)$ is said to be purely periodic modulo $m$. The smallest $\pi$ for which (4.1) is true is called the period of $\left(s_{n}\right)$ modulo $m$. All other $\pi$ 's are multiples of it.

The following theorem of Ward shows us how the period and rank are connected. 
Theorem 4.1. 26] Let $\left(h_{n}\right)$ be an EDS and $p$ be an odd prime whose rank of apparition $\rho$ is greater than 3 . Let $a_{1}$ be an integral solution of the congruence $a_{1} \equiv h_{2} / h_{\rho-2}(p)$ and let $e$ and $k$ be the exponents to which $a_{1}$ and $a_{2} \equiv h_{\rho-1}(p)$ respectively belong modulo $p$. Then $\left(h_{n}\right)$ is purely periodic modulo $p$, and its period $\pi$ is given by the formula $\pi\left(h_{n}\right)=\tau \rho$ where $\tau=2^{\nu}[e, k]$. Here $[e, k]$ is the least common multiple of $e$ and $k$, and the exponent $\nu$ is determined as follows:

$$
\nu=\left\{\begin{array}{cc}
+1 & \text { if } e \text { and } k \text { are both odd } \\
-1 & \text { if } e \text { and } k \text { are both even and both divisible by } \\
0 & \text { exactly the same power of } 2
\end{array}\right.
$$

We give the period of $\left(h_{n}\right)$ in the following theorem.

Theorem 4.2. Let $\left(h_{n}\right)$ be an elliptic divisibility sequence with $N$-th term zero, where $N \in\{4,5, \ldots, 10,12\}$ and let $p$ be an odd prime. Then the period of $\left(h_{n}\right)$ is

$$
\pi\left(h_{n}\right)= \begin{cases}t(p-1) & \text { if } q=[e, k] \text { is a primitive root modulo } p \\ 2 N l & \text { otherwise }\end{cases}
$$

where $l=\left\{\begin{array}{ll}q & \text { if } q \text { is odd } \\ q / 2 & \text { if } q \text { is even, }\end{array} \quad t= \begin{cases}N & \text { if } N \text { is even } \\ N / 2 & \text { if } N \text { is odd. }\end{cases}\right.$

Proof. The cases $N=4$ and 5 , can easily be seen, so we give $N=6$, other cases can be proved in similar way. In this case the period of $\left(h_{n}\right)$ is $\pi\left(h_{n}\right)=6(p-1)$ or 12l. By Theorem 3.1 and Theorem 4.1, we have $a_{1}=h_{2} / h_{4}=-1 / \alpha^{5}(\alpha+1)^{4}$ and $a_{2}=h_{5}=\alpha^{10}(\alpha+1)^{8}$. Let $e$ and $k$ be the orders of $a_{1}$ and $a_{2}$, respectively. Then $k=e / 2$ when $e$ is even, and $k=e$ when $e$ is odd, since $a_{2}=1 / a_{1}^{2}$. Let $a_{1}$ be a primitive root modulo $p$. Then $e=p-1, k=(p-1) / 2$ and so $q=p-1$. Hence $\nu=0$ and so $\tau=p-1$. Therefore in this case $\pi\left(h_{n}\right)=6(p-1)$. If $a_{1}$ is not a primitive root modulo $p$, then there are two cases. In the first case, let $q$ be odd. Then $e=k=q$, so that $\nu=1$. Thus $\tau=q$, therefore $\pi\left(h_{n}\right)=6 q$. In the second case, let $q$ be even. Then $e=q$ and $k=q / 2$, so that $\nu=0$. Thus $\tau=2 q$ therefore $\pi\left(h_{n}\right)=12 q$.

\begin{tabular}{|c|c|c|c|c|c|c|c|c|c|c|c|}
\hline$N=6$ & $\mathbb{F}_{5}$ & $\mathbb{F}_{7}$ & $\mathbb{F}_{11}$ & $\mathbb{F}_{13}$ & $\mathbb{F}_{17}$ & $\mathbb{F}_{19}$ & $\mathbb{F}_{23}$ & $\mathbb{F}_{29}$ & $\mathbb{F}_{31}$ & $\mathbb{F}_{37}$ & $\mathbb{F}_{41}$ \\
\hline$\alpha=-5$ & - & 36 & 60 & 72 & 96 & 108 & 132 & 84 & 180 & 72 & 120 \\
\hline$\alpha=-4$ & 12 & 12 & 60 & 36 & 12 & 108 & 132 & 84 & 180 & 36 & 12 \\
\hline$\alpha=-3$ & 24 & 36 & 60 & 12 & 96 & 36 & 12 & 168 & 180 & 108 & 240 \\
\hline$\alpha=-2$ & 24 & 36 & 12 & 72 & 48 & 108 & 132 & 168 & 12 & 216 & 24 \\
\hline$\alpha=1$ & 12 & 36 & 60 & 36 & 12 & 108 & 132 & 84 & 60 & 108 & 60 \\
\hline$\alpha=2$ & 24 & 36 & 60 & 24 & 48 & 36 & 132 & 168 & 180 & 216 & 24 \\
\hline$\alpha=3$ & 24 & 12 & 60 & 36 & 96 & 108 & 132 & 168 & 180 & 36 & 240 \\
\hline$\alpha=4$ & - & 36 & 60 & 36 & 12 & 108 & 132 & 12 & 36 & 36 & 60 \\
\hline$\alpha=5$ & - & 36 & 60 & 72 & 96 & 108 & 132 & 84 & 36 & 216 & 120 \\
\hline
\end{tabular}

The periods of the EDSs with rank six for some integer $\alpha$ parameter

The periods of the elliptic divisibility sequences for which the sixth term is zero modulo $p$ for some integer parameter $\alpha$ and $p>3$, appears in the tablo above. 


\section{SquARES AND CUBES IN EDSs}

The question of when a term of a Lucas sequence can be square has generated interest in the literature [2, 3, 18, 19]. Similar results concerning cubes were also obtained for specific sequences such as Fibonacci, Lucas and Pell numbers [16, 17. In [8, 9], we describe when a term of an elliptic divisibility sequence can be a square or a cube, if one of the first six terms is zero.

The ultimate purpose of this section is to determine square or cube terms in some special family of the elliptic divisibility sequences whose contain a zero term. In this section we determine square or cube terms of these sequences by using the general terms of them. In particular, we will investigate the answers of the following questions:

- Which terms of $\left(h_{n}\right)$ can be a square or a cube independent of $\alpha$ ? This question is answered for each case. For example, consider an elliptic divisibility sequence for which sixth term is zero,

$i$. if $n \equiv 1,5,7,11$ (12), then $h_{n}=\square$ for all $\alpha \in \mathbb{Z} \backslash\{-1,0\}$, ii. if $n \equiv 1,3,9,15,17$ (18), then $h_{n}=C$ for all $\alpha \in \mathbb{Z} \backslash\{-1,0\}$.

- Which terms of $\left(h_{n}\right)$ can not be a square or a cube? Starting with the fact that square or cube terms can be arise dependent on the parameter $\alpha$ it is seen that some terms of $\left(h_{n}\right)$ can not be a square or a cube for any choice of $\alpha$ for each case. For example, consider an elliptic divisibility sequence for which sixth term is zero,

$i$. if $n \equiv 2,3,9,10(12)$, then $h_{n}$ is not a square for all $\alpha \in \mathbb{Z} \backslash\{-1,0\}$, ii. if $n \equiv 2,5,7,11,13,16(18)$, then $h_{n}$ is not a cube for all $\alpha \in \mathbb{Z} \backslash\{-1,0\}$.

- Which terms of $\left(h_{n}\right)$ can be a square or a cube with admissible choice of $\alpha$ ? In addition to square or cube terms which determined in question one it is seen that a term of an EDS can be a square or a cube depending on the admissible choice of $\alpha$. For example, consider an elliptic divisibility sequence for which sixth term is zero,

$i$. if $n \equiv 4,8(12)$ then $h_{n}$ is a square iff $\alpha+1=\square$,

ii. if $n \equiv 4,14$ (18) then $h_{n}$ is a cube iff $\alpha+1=C$,

iii. if $n \equiv 8,10$ (18) then $h_{n}$ is a cube iff $\alpha=C$.

Especially when we look for the answers of our problems we deduce some equations whose solutions give the desired answers. The problem now reduces to establishing that Diophantine equations which are divided into five main classes, then finding all solutions of these equations and observing whether these solutions give the desired $\alpha$. It follows that observing the solutions of some Pell equations, classical equations, trivial equations and finding the integral points on elliptic curves with rank zero or one. Now we have many equations of similar type and so we make a table to bring together all of these equations and their solutions can observe from this chart. For example, consider an elliptic divisibility sequence for which the eighth term is zero. If $n \equiv 3,13(16)$, then we see that $h_{n}=\square$ iff

$$
(\alpha-1)(2 \alpha-1)=
$$

this leads to a Pell's equation $(4 \alpha-3)^{2}-8 \beta^{2}=1$ or a trivial equation $(4 \alpha-3)^{2}+$ $8 \beta^{2}=1$ where $\alpha, \beta$ are integers. Equations encountered in some cases turned into elliptic curves. In particular, if we have an elliptic curve with rank zero then the only integral points on this curve are the torsion points. These, in turn, can be computed by the Lutz-Nagell Theorem. If the elliptic curve has a rank different 
from zero then the Elliptic Logarithm Method is applied to find the all integral solutions. Throughout this paper the symbols $\square$ and $C$ mean a square and a cube of a non-zero rational number, i.e. $\square= \pm \beta^{2}$ where $\beta$ is an integer.

A basic observation is the following: For every equation, the distinct irreducible factors (over $\mathbb{Q}[\alpha]$ ) appearing in the left-hand side (if they are at least two) are pairwise relatively prime. ${ }^{1}$ This implies that, if the right hand-side is $\square$ (respectively, $C$ ), then every irreducible factor is $\square$ (respectively, $C$ ). We use of this fact, for a quite number of equations, it turns out that it is not necessary to make use of this fact for all irreducible factor of the left-hand side. ${ }^{2}$

Solutions of the equations

\begin{tabular}{|c|c|c|c|}
\hline Eq. No & $\begin{array}{c}\text { implies } \\
\text { or } \\
\text { is equivalent to }\end{array}$ & $\begin{array}{l}\text { is reduced to } \\
\text { or } \\
\text { is equivalent to }\end{array}$ & Comments \\
\hline 1 & $\alpha(\alpha+1)=\square$ & $(2 \alpha+1)^{2} \pm \beta^{2}=1$ & trivial eq. \\
\hline 5 & $\alpha(\alpha-1)=\square$ & $(2 \alpha-1)^{2} \pm \beta^{2}=1$ & trivial eq. \\
\hline $2,3,4$ & $\alpha=\beta_{1}^{3} \& \alpha+1=\beta_{2}^{3}$ & $\beta_{2}^{3}-\beta_{1}^{3}=1$ & trivial eq. \\
\hline $\begin{array}{c}6,10,15, \\
16,17,18, \\
19,29,30, \\
38,39,40 \\
41,42\end{array}$ & $\alpha=\beta_{1}^{3} \& \alpha-1=\beta_{2}^{3}$ & $\beta_{1}^{3}-\beta_{2}^{3}=1$ & trivial eq. \\
\hline 7 & $(\alpha-1)(2 \alpha-1)=\square$ & $\begin{array}{l}(4 \alpha-3)^{2}-8 \beta^{2}=1 \\
(4 \alpha-3)^{2}+8 \beta^{2}=1\end{array}$ & $\begin{array}{l}\text { Pell eq. } \\
\text { trivial eq. }\end{array}$ \\
\hline 8 & $\alpha(2 \alpha-1)=\square$ & $\begin{array}{l}(2 \alpha-1)^{2}-2 \beta^{2}=1 \\
(2 \alpha-1)^{2}+2 \beta^{2}=1\end{array}$ & $\begin{array}{l}\text { Pell eq. } \\
\text { trivial eq. }\end{array}$ \\
\hline $\begin{array}{c}11,25,47 \\
48,49\end{array}$ & $\alpha-1=\beta_{1}^{3} \& 2 \alpha-1=\beta_{2}^{3}$ & $\beta_{2}^{3}+2\left(-\beta_{1}^{3}\right)=1$ & $\begin{array}{l}\text { 'classical' } \\
\text { equation }{ }^{3}\end{array}$ \\
\hline 12 & $(\alpha-1)\left(\alpha^{2}-\alpha+1\right)=\square$ & $\alpha^{3}-2 \alpha^{2}+2 \alpha-1= \pm \beta^{2}$ & zero rank $^{4}$ \\
\hline 13 & $\alpha^{2}-\alpha+1=\square$ & $(2 \alpha-1)^{2} \pm \beta^{2}=-3$ & trivial eq. \\
\hline $14,20,21$ & $\alpha^{2}-\alpha+1=C$ & $\beta^{3}-48=(8 \alpha-4)^{2}$ & Ellog used ${ }^{5}$ \\
\hline 9 & $\alpha(\alpha-1)(2 \alpha-1)=\square$ & $\begin{array}{c}(2 \alpha)^{3}-3(2 \alpha)^{2}+2(2 \alpha)=\beta^{2} \\
(-2 \alpha)^{3}+3(-2 \alpha)^{2}+2(-2 \alpha)=\beta^{2}\end{array}$ & $\begin{array}{l}\text { zero } \text { rank }^{4} \\
\text { zero } \operatorname{rank}^{4}\end{array}$ \\
\hline 22 & $-\alpha^{2}+3 \alpha-1=\square$ & $(2 \alpha-3)^{2} \pm \beta^{2}=5$ & trivial eq. \\
\hline 23 & $(2 \alpha-1)\left(-\alpha^{2}+3 \alpha-1\right)=\square$ & $\begin{array}{c}(-2 \alpha)^{3}+7(-2 \alpha)^{2}+10(-2 \alpha)+4=\beta^{2} \\
(2 \alpha)^{3}-7(2 \alpha)^{2}+10(2 \alpha)-4=\beta^{2}\end{array}$ & $\begin{array}{l}\text { Ellog } \text { used }^{5} \\
\text { zero } \text { rank }^{4} \\
\end{array}$ \\
\hline $\begin{array}{l}24,26 \\
27,28 \\
\end{array}$ & $-\alpha^{2}+3 \alpha-1=C$ & $\beta^{3}+80=(8 \alpha-12)^{2}$ & Ellog used ${ }^{5}$ \\
\hline $31,45,46$ & $\alpha=\beta_{1}^{3} \& 2 \alpha-1=\beta_{2}^{3}$ & $\left(-\beta_{2}\right)^{3}+2 \beta_{1}^{3}=1$ & $\begin{array}{l}\text { 'classical' } \\
\text { equation }{ }^{3} \\
\end{array}$ \\
\hline 33 & $\begin{array}{l}\alpha(2 \alpha-1)\left(2 \alpha^{2}-2 \alpha+1\right) \\
\quad \times\left(3 \alpha^{2}-3 \alpha+1\right)=\square\end{array}$ & $\begin{array}{c}(3 \alpha)^{3}-3(3 \alpha)^{2}+3(3 \alpha)=\beta^{2} \\
\alpha(2 \alpha-1)<0\end{array}$ & $\begin{array}{l}\text { zero rank }^{4} \\
\text { trivial eq. }\end{array}$ \\
\hline
\end{tabular}


continued from previous page

\begin{tabular}{|c|c|c|c|}
\hline Eq. No & $\begin{array}{c}\text { implies } \\
\text { or } \\
\text { is equivalent to }\end{array}$ & $\begin{array}{l}\text { is reduced to } \\
\text { or } \\
\text { is equivalent to }\end{array}$ & Comments \\
\hline 34 & $(\alpha-1)\left(-2 \alpha^{2}+2 \alpha-1\right)=\square$ & $\begin{array}{c}(-2 \alpha)^{3}+4(-2 \alpha)^{2}+6(-2 \alpha)+4=\beta^{2} \\
(2 \alpha)^{3}-4(2 \alpha)^{2}+6(2 \alpha)-4=\beta^{2}\end{array}$ & $\begin{array}{l}\text { Ellog used } \\
\text { zero rank }\end{array}$ \\
\hline 32 & $3 \alpha^{2}-3 \alpha+1=\square$ & $\begin{array}{c}\beta^{2}-3(2 \alpha-1)^{2}=1 \\
\beta^{2}+3 \alpha^{2}=-1\end{array}$ & $\begin{array}{l}\text { Pell eq. } \\
\text { impossible }\end{array}$ \\
\hline 35,36 & $\alpha\left(3 \alpha^{2}-3 \alpha+1\right)=\square$ & $\begin{array}{c}(3 \alpha)^{3}-3(3 \alpha)^{2}+3(3 \alpha)=\beta^{2} \\
(-3 \alpha)^{3}+3(-3 \alpha)^{2}+3(-3 \alpha)=\beta^{2}\end{array}$ & $\begin{array}{l}\text { zero } \operatorname{rank}^{4} \\
\text { zero } \operatorname{rank}^{4}\end{array}$ \\
\hline 37 & $(2 \alpha-1)\left(-2 \alpha^{2}+2 \alpha-1\right)=\square$ & $\begin{array}{c}(4 \alpha)^{3}-6(4 \alpha)^{2}+16(4 \alpha)-16=\beta^{2} \\
(-4 \alpha)^{3}+6(-4 \alpha)^{2}+16(-4 \alpha)+16=\beta^{2}\end{array}$ & $\begin{array}{l}\text { zero } \text { rank }^{4} \\
\text { zero } \operatorname{rank}^{4}\end{array}$ \\
\hline 43,44 & $2 \alpha^{2}-2 \alpha+1=C$ & $\beta^{3}-4=(4 \alpha-2)^{2}$ & Ellog used $^{5}$ \\
\hline
\end{tabular}

Notes

${ }^{1}$ As a characteristic example, take equation 38. Firstly, $\alpha$ can not have a common prime factor $p$ with any of $\alpha-1,2 \alpha-1,2 \alpha^{2}-2 \alpha+1,3 \alpha^{2}-3 \alpha+1$. Indeed, $\alpha \equiv 0(p)$ implies that both $\alpha-1$ and $2 \alpha-1$ are $\equiv-1(p)$ and both $2 \alpha^{2}-2 \alpha+1$ and $3 \alpha^{2}-3 \alpha+1$ are $\equiv 1(p)$. Next, $\alpha-1$ can not have prime factor $p$ with any of $2 \alpha-1,2 \alpha^{2}-2 \alpha+1$, $3 \alpha^{2}-3 \alpha+1$, because $\alpha-1 \equiv 0(p)$ implies $\alpha \equiv 1(p)$ and, hence $2 \alpha^{2}-2 \alpha+1$ and $3 \alpha^{2}-3 \alpha+1$ are both $\equiv 1(p)$. Analogously, $2 \alpha-1$ can not have a common prime factor $p$ with neither $2 \alpha^{2}-2 \alpha+1$ nor $3 \alpha^{2}-3 \alpha+1$, because, $p$ should be odd, hence $\alpha \equiv 1 / 2(p)$ and, consequently $2 \alpha^{2}-2 \alpha+1 \equiv 1 / 2(p)$ and $3 \alpha^{2}-3 \alpha+1 \equiv 1 / 4(p)$. Finally, $2 \alpha^{2}-2 \alpha+1$ and $3 \alpha^{2}-3 \alpha+1$ are relatively prime because $3\left(2 \alpha^{2}-2 \alpha+1\right)-$ $2\left(3 \alpha^{2}-3 \alpha+1\right)=1$.

${ }^{2}$ For example, although equation 44 implies that all three $\alpha, 2 \alpha^{2}-2 \alpha+1,3 \alpha^{2}-3 \alpha+1$ are $C$, we only use the fact that the second one is $C$.

${ }^{3}$ The equation $x^{3}+2 y^{3}=1$ has the integer solution $(x, y)=(-1,1)$, hence, by Theorem 5, Chapter 24 of 14 can not have further solutions with $x y \neq 0$.

${ }^{4}$ The only solutions are those given by coordinates of the torsion points. These, in turn, can be computed by the Lutz-Nagell Theorem (see, for example, Corollary 7.2, Chapter VIII.7 of [21]); automatically, they can be calculated using e.g. the PARI-GP calculator [15] or the online MAGMA calculator [13].

${ }^{5}$ The Elliptic Logarithm Method is applied. This has been developed in 23. and, independently, in [7] and now is implemented in MAGMA [13]; see also [1].

5.1. The case $N=4$. In this part we will answer the question of when a term of an EDS for which the fourth term is zero can be a perfect square or a cube. Although the terms of the EDSs can be a square or a cube dependent only on the parameter $\alpha$, there are cases when square or cube terms independent of the any choice of $\alpha$. We first determine square or cube terms of the sequences for which the fourth term is zero in the following theorem.

Theorem 5.1. Let $\left(h_{n}\right)$ be an elliptic divisibility sequence for which the fourth term is zero. 
i. If $n \equiv 1,7$ (8), then $h_{n}=\square$ for all $\alpha \in \mathbb{Z} \backslash\{0\}$.

ii. If $n \equiv 1,3,5,7$ (8), then $h_{n}=C$ for all $\alpha \in \mathbb{Z} \backslash\{0\}$.

Proof. For $(i)$, if $n \equiv 1$ or 7 (8), then $n=8 k+1$ or $8 k+7(k \in \mathbb{N})$. Substituting these values into (3.2), we have

$$
h_{8 k+1}=\alpha^{24 k^{2}+6 k}, h_{8 k+7}=-\alpha^{24 k^{2}+42 k+18},
$$

respectively. Hence, $h_{n}=\square$ for all $\alpha \in \mathbb{Z} \backslash\{0\}$.

For $(i i)$, if $n \equiv 1,3,5$ or $7(8)$, then $n=8 k+1,8 k+3,8 k+5$ or $8 k+7(k \in \mathbb{N})$.

Putting these into (3.2), we have

$$
h_{8 k+1}=\alpha^{24 k^{2}+6 k}, h_{8 k+3}=-\alpha^{24 k^{2}+18 k+3}, h_{8 k+5}=\alpha^{24 k^{2}+30 k+9}
$$

and

$$
h_{8 k+7}=-\alpha^{24 k^{2}+42 k+18,}
$$

respectively. Therefore $h_{n}=C$ for all $\alpha \in \mathbb{Z} \backslash\{0\}$.

After having determined the square or cube terms independent of the any choice of $\alpha$, we will turn to cases when a term of an EDS for which the fourth term is zero can be a square or a cube dependent on the parameter $\alpha$ in the following theorem.

Theorem 5.2. Let $\left(h_{n}\right)$ be an elliptic divisibility sequence for which the fourth term is zero.

i. $h_{n}=\square$ for all $n \in \mathbb{N}$ iff $\alpha=\square$.

ii. $h_{n}=C$ for all $n \in \mathbb{N}$ iff $\alpha=C$.

Proof. For $(i)$, we have seen that if $n \equiv 1,7$ (8), then $h_{n}=\square$ for all $\alpha \in \mathbb{Z} \backslash\{0\}$ in the previous theorem. Now will see in which cases a term of $\left(h_{n}\right)$ can be a perfect square. Consider the case $n \equiv 5(8)$. Then $n=8 k+5$ for $k \in \mathbb{N}$. Putting this into (3.2), we have

$$
h_{8 k+5}=\alpha^{24 k^{2}+30 k+9} .
$$

Hence we have $\alpha=\square$ iff $h_{8 k+5}=\square$. The cases when $n \equiv 2,3,6$ (8) can be proved in the similar way. So, $\alpha=\square$ iff $h_{n}=\square$ for all $n \in \mathbb{N}$.

For (ii), we have also seen that if $n \equiv 1,3,5$ or $7(8)$, then $h_{n}=C$ for all $\alpha \in \mathbb{Z} \backslash\{0\}$ in the previous theorem. So we must consider the cases $n \equiv 2$ or 6 (8). Then we have

$$
h_{8 k+2}=-\alpha^{24 k^{2}+12 k+1} \text { and } h_{8 k+6}=\alpha^{24 k^{2}+36 k+13}
$$

for $k \in \mathbb{N}$, respectively, by (3.2). Hence we get $\alpha=C$ iff $h_{8 k+2}=C$ or $\alpha=C$ iff $h_{8 k+6}=C$. Therefore $\alpha=C$ iff $h_{n}=C$ for all $n \in \mathbb{N}$.

We can summarize the results which obtained above in the following table.

\begin{tabular}{||c|c|c|c|c|c|c|c|c||}
\hline \hline$N=4$ & $h_{8 k}$ & $h_{8 k+1}$ & $h_{8 k+2}$ & $h_{8 k+3}$ & $h_{8 k+4}$ & $h_{8 k+5}$ & $h_{8 k+6}$ & $h_{8 k+7}$ \\
\hline$\alpha \in \mathbb{Z} \backslash\{0\}$ & 0 & $\square$ & & & 0 & & & $\square$ \\
\hline$\alpha=\square$ & 0 & $\square$ & $\square$ & $\square$ & 0 & $\square$ & $\square$ & $\square$ \\
\hline$\alpha \in \mathbb{Z} \backslash\{0\}$ & 0 & $C$ & & $C$ & 0 & $C$ & & $C$ \\
\hline$\alpha=C$ & 0 & $C$ & $C$ & $C$ & 0 & $C$ & $C$ & $C$ \\
\hline \hline
\end{tabular}


5.2. The Case $N=5$. An easy calculation as in Theorem 5.1 and Theorem 5.2 gives the following theorems.

Theorem 5.3. Let $\left(h_{n}\right)$ be an elliptic divisibility sequence for which the fifth term is zero.

i. If $n \equiv 1,4,6,9$ (10), then $h_{n}=\square$ for all $\alpha \in \mathbb{Z} \backslash\{0\}$.

ii. If $n \equiv 1,3,4,11,12,14$ (15), then $h_{n}=C$ for all $\alpha \in \mathbb{Z} \backslash\{0\}$.

Theorem 5.4. Let $\left(h_{n}\right)$ be an elliptic divisibility sequence for which the fifth term is zero.

i. $h_{n}=\square$ for all $n \in \mathbb{N}$ iff $\alpha=\square$.

ii. $h_{n}=C$ for all $n \in \mathbb{N}$ iff $\alpha=C$.

5.3. The Case $N=6$. This case is little more complicated than the other ones. We determine the square or cube terms dependent on the any choice of $\alpha$ and we also determine the square or cube terms dependent on the admissible choice of $\alpha$ in the following theorems. An easy calculation as in Theorem 5.1 and Theorem 5.2 gives the following theorems.

Theorem 5.5. Let $\left(h_{n}\right)$ be an elliptic divisibility sequence for which the sixth term is zero.

i. If $n \equiv 1,5,7,11$ (12), then $h_{n}=\square$ for all $\alpha \in \mathbb{Z} \backslash\{-1,0\}$.

ii. If $n \equiv 1,3,9,15,17$ (18), then $h_{n}=C$ for all $\alpha \in \mathbb{Z} \backslash\{-1,0\}$.

Theorem 5.6. Let $\left(h_{n}\right)$ be an elliptic divisibility sequence for which the sixth term is zero.

i. If $n \equiv 4,8$ (12), $\alpha+1=\square$ iff $h_{n}=\square$.

ii. If $n \equiv 4,14(18), \alpha+1=C$ iff $h_{n}=C$.

iii. If $n \equiv 8,10$ (18), $\alpha=C$ iff $h_{n}=C$.

It is shown that the terms of the EDS for which the sixth term is zero can not be a square or a cube for any choice of $\alpha$ in the following theorem.

Theorem 5.7. Let $\left(h_{n}\right)$ be an elliptic divisibility sequence for which the sixth term is zero.

i. If $n \equiv 2,3,9,10(12)$, then $h_{n}$ is not a square for all $\alpha \in \mathbb{Z} \backslash\{-1,0\}$.

ii. If $n \equiv 2,5,7,11,13,16(18)$, then $h_{n}$ is not a cube for all $\alpha \in \mathbb{Z} \backslash\{-1,0\}$.

Proof. For $(i)$, if $n \equiv 2(12)$ then $n=12 k+2(k \in \mathbb{N})$. Substituting this into (3.4), we have

$$
h_{12 k+2}=-\alpha^{60 k^{2}+20 k+1}(\alpha+1)^{48 k^{2}+16 k+1} .
$$

Therefore we have $h_{n}=\square$ iff

$$
\alpha(\alpha+1)=\square .
$$

This last equation leads to trivial equation

$$
(2 \alpha+1)^{2} \pm \beta^{2}=1
$$

where $\beta$ is an integer. It is clear that the solutions of these equations do not provide desired $\alpha$. The cases where $n \equiv 3,9,10(12)$ can be proved in the same way.

For $(i i)$, if $n \equiv 2$ or $16(18)$ then $n=18 k+2$ or $n=18 k+16(k \in \mathbb{N})$. Putting these into (3.4), we have

$$
h_{18 k+2}=-\alpha^{135 k^{2}+30 k+1}(\alpha+1)^{108 k^{2}+24 k+1}
$$


and

$$
h_{18 k+16}=\alpha^{135 k^{2}+240 k+106}(\alpha+1)^{108 k^{2}+192 k+85}
$$

respectively. Thus $h_{n}=C$ iff

$$
\alpha(\alpha+1)=C .
$$

If $n \equiv 5$ or 13 (18) then we have

$$
h_{18 k+5}=\alpha^{135 k^{2}+75 k+10}(\alpha+1)^{108 k^{2}+60 k+8}
$$

and

$$
h_{18 k+13}=\alpha^{135 k^{2}+195 k+70}(\alpha+1)^{108 k^{2}+156 k+56}
$$

respectively, by (3.4). From these equations we see that $h_{n}=C$ iff

$$
\alpha(\alpha+1)^{2}=C .
$$

Now suppose that $n \equiv 7$ or 11 (18) then we have

$$
h_{18 k+7}=\alpha^{135 k^{2}+105 k+20}(\alpha+1)^{108 k^{2}+84 k+16}
$$

and

$$
h_{18 k+11}=-\alpha^{135 k^{2}+165 k+50}(\alpha+1)^{108 k^{2}+132 k+40}
$$

respectively, by (3.4). In this case we have $h_{n}=C$ iff

$$
\alpha^{2}(\alpha+1)=C .
$$

It follows that equations (2),(3) and (4) lead to trivial equation

$$
\beta_{2}^{3}-\beta_{1}^{3}=1
$$

where $\alpha=\beta_{1}^{3}, \alpha+1=\beta_{2}^{3}$ and $\beta_{1}, \beta_{2}$ are integers. Solutions of this equation do not provide desired $\alpha$.

5.4. The Case $N=7$. An easy calculation as in Theorem 5.1 and Theorem 5.2 gives the following theorems.

Theorem 5.8. Let $\left(h_{n}\right)$ be an elliptic divisibility sequence for which the seventh term is zero.

i. If $n \equiv 1,13$ (14), then $h_{n}=\square$ for all $\alpha \in \mathbb{Z} \backslash\{0,1\}$.

ii. If $n \equiv 1,3,8,13,18,20$ (21), then $h_{n}=C$ for all $\alpha \in \mathbb{Z} \backslash\{0,1\}$.

Theorem 5.9. Let $\left(h_{n}\right)$ be an elliptic divisibility sequence for which the seventh term is zero.

i. If $n \equiv 2,3,11,12(14)$, then $h_{n}=\square$ iff $\alpha-1=\square$.

ii. If $n \equiv 4,5,9,10$ (14), then $h_{n}=\square$ iff $\alpha=\square$.

iii. If $n \equiv 4,6,10,11,15,17(21)$, then $h_{n}=C$ iff $\alpha=C$.

iv. If $n \equiv 9,12(21)$, then $h_{n}=C$ iff $\alpha-1=C$.

Theorem 5.10. Let $\left(h_{n}\right)$ be an elliptic divisibility sequence for which the seventh term is zero.

i. If $n \equiv 6,8$ (14) then $h_{n}$ is not a square for all $\alpha \in \mathbb{Z} \backslash\{0,1\}$.

ii. If $n \equiv 2,5,16,19$ (21) then $h_{n}$ is not a cube for all $\alpha \in \mathbb{Z} \backslash\{0,1\}$. 
Proof. For $(i)$, if $n \equiv 6$ or 8 (14) then we have

$$
h_{14 k+6}=-\alpha^{140 k^{2}+120 k+1}(\alpha-1)^{84 k^{2}+72 k+15}
$$

and

$$
h_{14 k+8}=\alpha^{140 k^{2}+160 k+45}(\alpha-1)^{84 k^{2}+96 k+27}
$$

respectively, by (3.5). From these equations we see that $h_{n}=\square$ iff

$$
\alpha(\alpha-1)=\square .
$$

This last equation leads to trivial equations

$$
(2 \alpha-1)^{2} \pm \beta^{2}=1
$$

where $\beta$ is an integer. It is clear that the solutions of these equations do not provide desired $\alpha$.

For $(i i)$, if $n \equiv 2,5,16$ or 19 (21) then we have

$$
\begin{aligned}
h_{21 k+2} & =-\alpha^{315 k^{2}+60 k+2}(\alpha-1)^{189 k^{2}+36 k+1} \\
h_{21 k+5} & =\alpha^{315 k^{2}+150 k+17}(\alpha-1)^{189 k^{2}+90 k+10} \\
h_{21 k+16} & =-\alpha^{315 k^{2}+480 k+182}(\alpha-1)^{189 k^{2}+288 k+109} \\
h_{21 k+19} & =\alpha^{315 k^{2}+570 k+257}(\alpha-1)^{189 k^{2}+342 k+154}
\end{aligned}
$$

respectively, by (3.5). Therefore, $h_{n}=C$ iff

$$
\alpha^{2}(\alpha-1)=C .
$$

This last equation leads to trivial equation

$$
\beta_{1}^{3}-\beta_{2}^{3}=1
$$

where $\alpha=\beta_{1}^{3}, \alpha-1=\beta_{2}^{3}$ and $\beta_{1}, \beta_{2}$ are integers and solutions of this equation do not provide desired $\alpha$.

5.5. The Case $N=8$. We determine square or cube terms dependent on the any choice of $\alpha$ and independent of the admissible choice of $\alpha$ in the following theorem.

Theorem 5.11. Let $\left(h_{n}\right)$ be an elliptic divisibility sequence for which the eighth term is zero.

i. If $n \equiv 1,4,12,15$ (16), then $h_{n}=\square$ for all $\alpha \in \mathbb{Z} \backslash\{0,1\}$.

ii. If $n \equiv 3,13(16)$, then $h_{n}=\square$ iff $(\alpha-1)(2 \alpha-1)=\square$.

iii. If $n \equiv 5,11(16)$, then $h_{n}=\square$ iff $\alpha(2 \alpha-1)=\square$.

Proof. For $(i)$, if $n \equiv 1,4,12$ or 15 (16), then $n=16 k+1, n=16 k+4, n=16 k+12$, or $16 k+15(k \in \mathbb{N})$. Substituting these values into (3.6), we have

$$
\begin{gathered}
h_{16 k+1}=\alpha^{240 k^{2}+30 k}(\alpha-1)^{112 k^{2}+14 k}(2 \alpha-1)^{96 k^{2}+12 k}, \\
h_{16 k+4}=\alpha^{240 k^{2}+120 k+4}(\alpha-1)^{112 k^{2}+56 k+6}(2 \alpha-1)^{96 k^{2}+48 k+6}, \\
h_{16 k+12}=-\alpha^{240 k^{2}+360 k+134}(\alpha-1)^{112 k^{2}+168 k+62}(2 \alpha-1)^{96 k^{2}+144 k+54},
\end{gathered}
$$

and

$$
h_{16 k+15}=-\alpha^{240 k^{2}+450 k+134}(\alpha-1)^{112 k^{2}+210 k+98}(2 \alpha-1)^{96 k^{2}+180 k+84},
$$

respectively. Hence, $h_{n}=\square$ for all $\alpha \in \mathbb{Z} \backslash\{0,1\}$.

For $(i i)$, if $n \equiv 3,13$ (16) then we have

$$
h_{16 k+3}=-\alpha^{240 k^{2}+90 k+8}(\alpha-1)^{112 k^{2}+42 k+3}(2 \alpha-1)^{96 k^{2}+36 k+3}
$$


and

$$
h_{16 k+13}=\alpha^{240 k^{2}+390 k+158}(\alpha-1)^{112 k^{2}+182 k+73}(2 \alpha-1)^{96 k^{2}+156 k+63},
$$

respectively by (3.6). Hence, $h_{n}=\square$ iff

$$
(\alpha-1)(2 \alpha-1)=\square .
$$

It follows that

$$
(4 \alpha-3)^{2}-8 \beta^{2}=1
$$

Or

$$
(4 \alpha-3)^{2}+8 \beta^{2}=1
$$

where $\beta$ is an integer. From the last equation we have no solutions of $\alpha$. The first equation leads to Pell equation. If we rewrite this equation as

$$
(\tau+2 \beta \sqrt{2})(\tau-2 \beta \sqrt{2})=1
$$

where $\tau=4 \alpha-3$ we see that the only solutions of the form $\tau_{k}+3 \equiv 0$ (4) give the desired solution of $\alpha$ and their number is infinite.

For $(i i i)$, if $n \equiv 5$ or 11 (16) then we have

$$
h_{16 k+5}=\alpha^{240 k^{2}+150 k+23}(\alpha-1)^{112 k^{2}+70 k+10}(2 \alpha-1)^{96 k^{2}+60 k+9}
$$

and

$$
h_{16 k+11}=-\alpha^{240 k^{2}+330 k+113}(\alpha-1)^{112 k^{2}+154 k+52}(2 \alpha-1)^{96 k^{2}+132 k+45},
$$

respectively, by (3.6). Hence, $h_{n}=\square$ iff

$$
\alpha(2 \alpha-1)=\square .
$$

It follows that $(\alpha, 2 \alpha-1)=\left(\beta_{1}^{2}, \beta_{2}^{2}\right),\left(-\beta_{1}^{2},-\beta_{2}^{2}\right),\left(-\beta_{1}^{2}, \beta_{2}^{2}\right)$ or $\left(\beta_{1}^{2},-\beta_{2}^{2}\right)$, where $\beta_{1}, \beta_{2}$ are positive integers. The latter two possibilities give the trivial equations $2 \beta_{1}^{2}+\beta_{2}^{2}=-1$ and $2 \beta_{1}^{2}+\beta_{2}^{2}=1$, respectively. The first equation is impossible and the second one does not give desired $\alpha$. The former two possibilities lead to Pell equations

$$
\begin{aligned}
& \beta_{2}^{2}-2 \beta_{1}^{2}=-1 \\
& \beta_{2}^{2}-2 \beta_{1}^{2}=1
\end{aligned}
$$

respectively. The solutions to the first equation are $(1,1),(7,5),(41,29), \ldots$ and parameters $\alpha$ corresponding to these solutions are $1,25,841, \ldots$. Note that $\alpha$ can not be 1 by the assumption. The solutions to the last equation are $(3,2),(17,12),(99,70), \ldots$ and parameters $\alpha$ corresponding to these solutions are $-4,-144,-4900, \ldots$.

It is shown that the terms of the EDS for which the eighth term is zero can not be a square for any choice of $\alpha$ in the following theorem.

Theorem 5.12. Let $\left(h_{n}\right)$ be an elliptic divisibility sequence for which the eighth term is zero. If $n \equiv 2,6,7,9,10,14$ (16) then $h_{n}$ is not a square for all $\alpha \in \mathbb{Z} \backslash\{0,1\}$.

Proof. If $n \equiv 2,6,10$ or 14 (16) then we have

$$
\begin{gathered}
h_{16 k+2}=-\alpha^{240 k^{2}+60 k+3}(\alpha-1)^{112 k^{2}+28 k+1}(2 \alpha-1)^{96 k^{2}+24 k+1}, \\
h_{16 k+6}=-\alpha^{240 k^{2}+180 k+33}(\alpha-1)^{112 k^{2}+84 k+15}(2 \alpha-1)^{96 k^{2}+72 k+13}, \\
h_{16 k+10}=\alpha^{240 k^{2}+420 k+93}(\alpha-1)^{112 k^{2}+140 k+43}(2 \alpha-1)^{96 k^{2}+120 k+37},
\end{gathered}
$$


and

$$
h_{16 k+14}=\alpha^{240 k^{2}+420 k+183}(\alpha-1)^{112 k^{2}+196 k+85}(2 \alpha-1)^{96 k^{2}+168 k+73},
$$

respectively, by (3.6). From these equations we see that $h_{n}=\square$ iff

$$
\alpha(\alpha-1)(2 \alpha-1)=\square,
$$

or equivalently $h_{n}=\square$ iff

$$
(2 \alpha)^{3}-3(2 \alpha)^{2}+2(2 \alpha)=\beta^{2}
$$

or

$$
(-2 \alpha)^{3}+3(-2 \alpha)^{2}+2(-2 \alpha)=\beta^{2} .
$$

These last equations give elliptic curves with rank 0 and torsion points on the these curves do not provide desired solutions of $\alpha$.

Let $n \equiv 7,9$ (16). Then we have

$$
h_{16 k+7}=-\alpha^{240 k^{2}+210 k+45}(\alpha-1)^{112 k^{2}+98 k+21}(2 \alpha-1)^{96 k^{2}+84 k+18},
$$

and

$$
h_{16 k+9}=\alpha^{240 k^{2}+270 k+75}(\alpha-1)^{112 k^{2}+126 k+35}(2 \alpha-1)^{96 k^{2}+108 k+30},
$$

respectively, by (3.6). From these equations we see that $h_{n}=\square$ iff

$$
\alpha(\alpha-1)=\square .
$$

This equation give trivial equations $(2 \alpha-1)^{2} \pm \beta^{2}=1$. It is clear that the solutions of these equations do not provide desired solutions of $\alpha$.

We give cube terms independent of the admissible choice of $\alpha$ in the following theorem.

Theorem 5.13. Let $\left(h_{n}\right)$ be an elliptic divisibility sequence for which the eighth term is zero.

i. If $n \equiv 1,7,17,23(24)$, then $h_{n}=C$ for all $\alpha \in \mathbb{Z} \backslash\{0,1\}$.

ii. If $n \equiv 3,4,20,21(24)$, then $h_{n}=C$ iff $\alpha=C$.

iii. If $n \equiv 6,18$ (24), then $h_{n}=C$ iff $2 \alpha-1=C$.

iv. If $n \equiv 9,15$ (24), then $h_{n}=C$ iff $\alpha-1=C$.

Proof. For $(i)$, if $n \equiv 1,7,17$ or $23(24)$, then $n=24 k+1, n=24 k+7, n=24 k+17$, or $24 k+23(k \in \mathbb{N})$. Substituting these values into (3.6), we have

$$
\begin{gathered}
h_{24 k+1}=\alpha^{540 k^{2}+45 k}(\alpha-1)^{252 k^{2}+21 k}(2 \alpha-1)^{216 k^{2}+18 k}, \\
h_{24 k+7}=-\alpha^{540 k^{2}+315 k+45}(\alpha-1)^{252 k^{2}+147 k+21}(2 \alpha-1)^{216 k^{2}+126 k+18}, \\
h_{24 k+17}=\alpha^{540 k^{2}+765 k+270}(\alpha-1)^{252 k^{2}+357 k+126}(2 \alpha-1)^{216 k^{2}+306 k+108},
\end{gathered}
$$

and

$$
h_{24 k+23}=-\alpha^{540 k^{2}+1035 k+495}(\alpha-1)^{252 k^{2}+483 k+231}(2 \alpha-1)^{216 k^{2}+414 k+198},
$$

respectively. Hence, $h_{n}=C$ for all $\alpha \in \mathbb{Z} \backslash\{0,1\}$.

For $(i i)$, if $n \equiv 3,4,20$ or 21 (24), then $n=24 k+3, n=24 k+4, n=24 k+20$, or $24 k+21(k \in \mathbb{N})$. Substituting these values into (3.6), we have

$$
\begin{gathered}
h_{24 k+3}=-\alpha^{540 k^{2}+135 k+8}(\alpha-1)^{252 k^{2}+63 k+3}(2 \alpha-1)^{216 k^{2}+54 k+3}, \\
h_{24 k+4}=-\alpha^{540 k^{2}+180 k+14}(\alpha-1)^{252 k^{2}+84 k+6}(2 \alpha-1)^{216 k^{2}+72 k+6}, \\
h_{24 k+20}=-\alpha^{540 k^{2}+900 k+374}(\alpha-1)^{252 k^{2}+420 k+174}(2 \alpha-1)^{216 k^{2}+360 k+150},
\end{gathered}
$$


and

$$
h_{24 k+21}=\alpha^{540 k^{2}+945 k+413}(\alpha-1)^{252 k^{2}+441 k+192}(2 \alpha-1)^{216 k^{2}+378 k+165},
$$

respectively. Thus $h_{n}=C$ iff $\alpha^{2}=C$. The cases (iii) and (iv) can be proved in the same way.

It is shown that the terms of the EDS for which the eighth term is zero can not be a cube for any choice of $\alpha$ in the following theorem.

Theorem 5.14. Let $\left(h_{n}\right)$ be an elliptic divisibility sequence for which the eighth term is zero. If $n \equiv 2,5,10,11,12,13,14,19,22(24)$ then $h_{n} \neq C$ for all $\alpha \in \mathbb{Z} \backslash\{0,1\}$.

Proof. If $n \equiv 5$ (24) then

$$
h_{24 k+5}=\alpha^{540 k^{2}+225 k+23}(\alpha-1)^{252 k^{2}+105 k+10}(2 \alpha-1)^{216 k^{2}+90 k+9}
$$

by (3.6). So, $h_{n}=C$ iff

$$
\alpha^{2}(\alpha-1)=C
$$

Let $n \equiv 12(24)$. Then we have

$$
h_{24 k+12}=\alpha^{540 k^{2}+540 k+134}(\alpha-1)^{252 k^{2}+252 k+62}(2 \alpha-1)^{216 k^{2}+216 k+54},
$$

by (3.6). Therefore $h_{n}=C$ iff

$$
\alpha^{2}(\alpha-1)^{2}=C
$$

The equations (6) and (10) lead to trivial equation

$$
\beta_{1}^{3}-\beta_{2}^{3}=1
$$

where $\alpha=\beta_{1}^{3}, \alpha-1=\beta_{2}^{3}$ and $\beta_{1}, \beta_{2}$ are integers and solutions of this equation do not provide desired $\alpha$. The cases where $n \equiv 11,13,19$ (24) can be proved in the same way.

If $n \equiv 2,10,14,22(24)$ then

$$
\begin{gathered}
h_{24 k+2}=\alpha^{540 k^{2}+90 k+3}(\alpha-1)^{252 k^{2}+42 k+1}(2 \alpha-1)^{216 k^{2}+36 k+1}, \\
h_{24 k+10}=-\alpha^{540 k^{2}+450 k+93}(\alpha-1)^{252 k^{2}+210 k+43}(2 \alpha-1)^{216 k^{2}+180 k+37}, \\
h_{24 k+14}=-\alpha^{540 k^{2}+630 k+183}(\alpha-1)^{252 k^{2}+294 k+85}(2 \alpha-1)^{216 k^{2}+252 k+73}, \\
h_{24 k+22}=\alpha^{540 k^{2}+990 k+453}(\alpha-1)^{252 k^{2}+462 k+211}(2 \alpha-1)^{216 k^{2}+396 k+181},
\end{gathered}
$$

respectively, by (3.6). From these equations we see that $h_{n}=C$ iff

$$
(\alpha-1)(2 \alpha-1)=C .
$$

It follows that $(\alpha-1,2 \alpha-1)=\left(\beta_{1}^{3}, \beta_{2}^{3}\right)$ where $\beta_{1}, \beta_{2}$ are integers. This gives the classical equation

$$
\beta_{2}^{3}+2\left(-\beta_{1}\right)^{3}=1
$$

and the only solution of this equation is $\left(\beta_{1}, \beta_{2}\right)=(-1,-1)$ and this solution does not provide desired $\alpha$. 
5.6. The Case $N=9$. We determine square terms independent of the admissible choice of $\alpha$. An easy calculation as in Theorem 5.1 and Theorem 5.2 gives the following theorems.

Theorem 5.15. Let $\left(h_{n}\right)$ be an elliptic divisibility sequence for which the ninth term is zero.

i. If $n \equiv 1,17$ (18), then $h_{n}=\square$ for all $\alpha \in \mathbb{Z} \backslash\{0,1\}$.

ii. If $n \equiv 5,13$ (18), then $h_{n}=\square$ iff $\alpha=\square$.

It is shown that the terms of the EDS for which the ninth term is zero can not be a square for any choice of $\alpha$ in the following theorem.

Theorem 5.16. Let $\left(h_{n}\right)$ be an elliptic divisibility sequence for which the ninth term is zero. If $n \equiv 2,3,4,6,7,8,10,11,12,14,15,16$ (18) then $h_{n}$ is not a square for all $\alpha \in \mathbb{Z} \backslash\{0,1\}$.

Proof. If $n \equiv 6,7,8,10,11$ or 12 (18) then $h_{n}=\square$ iff

$$
\alpha(\alpha-1)=
$$

by (3.7). But this is impossible by proof of Theorem 5.10 .

If $n \equiv 2,3,15,16$ (18) then $h_{n}=\square$ iff

$$
(\alpha-1)\left(\alpha^{2}-\alpha+1\right)=
$$

by (3.7), or equivalently $h_{n}=\square$ iff $\alpha^{3}-2 \alpha^{2}+2 \alpha-1= \pm \beta^{2}$ where $\beta$ is an integer. These last equations give elliptic curves with rank 0 and torsion points on these curves do not give desired $\alpha$.

Let $n \equiv 4,14$ (18). Then $h_{n}=\square$ iff

$$
\alpha^{2}-\alpha+1=
$$

by (3.7). This last equation leads to trivial equations

$$
(2 \alpha-1)^{2}-\beta^{2}=-3
$$

or

$$
(2 \alpha-1)^{2}+\beta^{2}=-3
$$

where $\beta$ is an integer. The last equation is impossible and from the first one we only have $\alpha=0$ and 1 .

We give the cube terms in the following theorem.

Theorem 5.17. Let $\left(h_{n}\right)$ be an elliptic divisibility sequence for which the ninth term is zero.

i. If $n \equiv 1,3,6,12,15,21,24,26(27)$, then $h_{n}=C$ for all $\alpha \in \mathbb{Z} \backslash\{0,1\}$.

ii. If $n \equiv 4,23(27)$, then $h_{n}=C$ iff $\left(\alpha^{2}-\alpha+1\right)^{2}=C$.

Proof. For $(i)$, if $n \equiv 1,3,6,12,15,21,24,26$ (27), then an easy calculation gives that $h_{n}=C$ for all $\alpha \in \mathbb{Z} \backslash\{0,1\}$.

For $(i i)$, if $n \equiv 4,23(27)$, then $h_{n}=C$ iff

$$
\left(\alpha^{2}-\alpha+1\right)^{2}=C
$$

or equivalently $\alpha^{2}-\alpha+1=C$ by (3.7). This last equation leads to an elliptic curve with rank 1

$$
\beta^{3}-48=(8 \alpha-4)^{2}
$$

where $\beta$ is an integer. Applying the Elliptic Logarithm Method we see that the only solutions to the above equation are $\alpha=-18$ and 19 . 
Theorem 5.18. Let $\left(h_{n}\right)$ be an elliptic divisibility sequence for which the ninth term is zero. If $n \equiv 2,5,7,8,10,11,13,14,16,17,19,20,22,25$ (27) then $h_{n} \neq C$ for all $\alpha \in \mathbb{Z} \backslash\{0,1\}$.

Proof. If $n \equiv 8,19$ (27), then $h_{n}=C$ iff

$$
\alpha(\alpha-1)=C,
$$

if $n \equiv 10,17(27)$, then $h_{n}=C$ iff

$$
\alpha^{2}(\alpha-1)^{2}=C
$$

if $n \equiv 2,25(27)$, then $h_{n}=C$ iff

$$
\alpha^{2}(\alpha-1)\left(\alpha^{2}-\alpha+1\right)=C,
$$

if $n \equiv 5,22(27)$, then $h_{n}=C$ iff

$$
\alpha(\alpha-1)\left(\alpha^{2}-\alpha+1\right)^{2}=C,
$$

and if $n \equiv 13,14(27)$, then $h_{n}=C$ iff

$$
\alpha^{2}(\alpha-1)^{2}\left(\alpha^{2}-\alpha+1\right)^{2}=C .
$$

respectively by (3.7). These equations lead to trivial equation

$$
\beta_{1}^{3}-\beta_{2}^{3}=1
$$

where $\alpha=\beta_{1}^{3}, \alpha-1=\beta_{2}^{3}$ and $\beta_{1}, \beta_{2}$ are integers. It is clear that the solutions of this equation do not provide desired $\alpha$.

If $n \equiv 7,20(27)$ then $h_{n}=C$ iff

$$
\alpha\left(\alpha^{2}-\alpha+1\right)=C,
$$

and if $n \equiv 11,16(27)$ then $h_{n}=C$ iff

$$
(\alpha-1)^{2}\left(\alpha^{2}-\alpha+1\right)=C
$$

respectively by (3.7). These equations lead to

$$
\beta^{3}-48=(8 \alpha-4)^{2}
$$

where $\beta$ is an integer. It is clear that the solutions of this equation do not provide desired $\alpha$.

5.7. The Case $N=10$. We determine square terms in the following theorem.

Theorem 5.19. Let $\left(h_{n}\right)$ be an elliptic divisibility sequence for which the tenth term is zero.

i. If $n \equiv 1,9,11,19$ (20), then $h_{n}=\square$ for all $\alpha \in \mathbb{Z} \backslash\{0,1\}$.

ii. If $n \equiv 4,16(20)$, then $h_{n}=\square$ iff $-\alpha^{2}+3 \alpha-1=\square$.

iii. If $n \equiv 5,15$ (20), then $h_{n}=\square$ iff $\alpha=\square$.

Proof. The cases $(i)$ and (iii) can be proved in the same way as in Theorems 5.1 and 5.2. If $n \equiv 4,16(20)$, then $h_{n}=\square$ iff

$$
-\alpha^{2}+3 \alpha-1=
$$

by (3.8). This equation leads to trivial equations

$$
(2 \alpha-3)^{2} \pm \beta^{2}=5
$$

where $\beta$ is an integer. The only solutions of these equations are $\alpha=2$ and 3 . 
It is shown that the terms of the EDS for which the tenth term is zero can not be a square for any choice of $\alpha$ in the following theorem.

Theorem 5.20. Let $\left(h_{n}\right)$ be an elliptic divisibility sequence for which the tenth term is zero. If $n \equiv 2,3,6,7,8,12,13,14,17,18(20)$, then $h_{n}$ is not a square for all $\alpha \in \mathbb{Z} \backslash\{0,1\}$.

Proof. If $n \equiv 2,3,7,13,17,18(20)$, then $h_{n}=\square$ iff

$$
\alpha(\alpha-1)(2 \alpha-1)=
$$

by (3.8). But this is impossible by proof of Theorem 5.12 .

If $n \equiv 6,14(20)$, then $h_{n}=\square$ iff

$$
\alpha(\alpha-1)=
$$

by (3.8), but this is impossible by proof of Theorem 5.10 .

If $n \equiv 8,12(20)$, then $h_{n}=\square$ iff

$$
(2 \alpha-1)\left(-\alpha^{2}+3 \alpha-1\right)=
$$

by (3.8). This last equation leads to

$$
(2 \alpha)^{3}-7(2 \alpha)^{2}+10(2 \alpha)-4=\beta^{2}
$$

or

$$
(-2 \alpha)^{3}+7(-2 \alpha)^{2}+10(-2 \alpha)+4=\beta^{2}
$$

where $\beta$ is an integer. From the first equation we have an elliptic curve with rank zero and the torsion points on this curve do not provide desired $\alpha$. From the last equation we have an elliptic curve with rank 1 and applying the Elliptic Logarithm Method we see that the integral points on this curve do not give desired $\alpha$.

We give cube terms in the following theorem.

Theorem 5.21. Let $\left(h_{n}\right)$ be an elliptic divisibility sequence for which the tenth term is zero.

i. If $n \equiv 1,11,19,29(30)$, then $h_{n}=C$.

ii. If $n \equiv 3,27$ (30), then $h_{n}=C$ iff $-\alpha^{2}+3 \alpha-1=C$.

iii. If $n \equiv 7,13,17,23(30)$, then $h_{n}=C$ iff $2 \alpha-1=C$.

Proof. The cases $(i)$ and (iii) can be proved in the same way as in Theorems 5.1 and 5.2. If $n \equiv 3,27(30)$, then $h_{n}=C$ iff

$$
-\alpha^{2}+3 \alpha-1=C
$$

by (3.8). This equation leads to

$$
\beta^{3}+80=(8 \alpha-12)^{2}
$$

where $\beta$ is an integer. From this equation we have an elliptic curve with rank 1 and applying the Elliptic Logarithm Method we see that the only solutions to this equation are $\alpha=2,3,38$ and -35 .

Theorem 5.22. Let $\left(h_{n}\right)$ be an elliptic divisibility sequence for which the tenth term is zero. If $n \equiv 2,4,5,6,8,9,12,14,15,16,18,21,22,24,25,26,28$ (30) then $h_{n} \neq C$ for all $\alpha \in \mathbb{Z} \backslash\{0,1\}$. 
Proof. If $n \equiv 2,8,22,28(30)$, then $h_{n}=C$ iff

$$
(\alpha-1)(2 \alpha-1)\left(-\alpha^{2}+3 \alpha-1\right)=C
$$

by (3.8). This equation leads to classical equation

$$
\beta_{2}^{3}+2\left(-\beta_{1}^{3}\right)=1
$$

where $\alpha-1=\beta_{1}^{3}, 2 \alpha-1=\beta_{2}^{3}$ and $\beta_{1}, \beta_{2}$ are integers and the only solution of this equation is $\left(\beta_{1}, \beta_{2}\right)=(-1,-1)$ and this solution does not provide desired $\alpha$.

If $n \equiv 4,14,16,26(30)$, then $h_{n}=C$ iff

$$
\alpha\left(-\alpha^{2}+3 \alpha-1\right)=C,
$$

if $n \equiv 9,21(30)$, then $h_{n}=C$ iff

$$
(2 \alpha-1)^{2}\left(-\alpha^{2}+3 \alpha-1\right)=C,
$$

if $n \equiv 12,18(30)$, then $h_{n}=C$ iff

$$
(\alpha-1)\left(-\alpha^{2}+3 \alpha-1\right)^{2}=C,
$$

by (3.8) or equivalently $\alpha^{2}-3 \alpha+1=C$. This last equation leads to equation (5.2). The solutions of this equation do not provide desired $\alpha$.

If $n \equiv 5,25(30)$, then $h_{n}=C$ iff

$$
\alpha(\alpha-1)(2 \alpha-1)=C,
$$

if $n \equiv 15(30)$, then $h_{n}=C$ iff

$$
\alpha(\alpha-1)\left(-\alpha^{2}+3 \alpha-1\right)=C .
$$

by (3.8). These equations leads to trivial equation

$$
\beta_{1}^{3}-\beta_{2}^{3}=1
$$

where $\alpha=\beta_{1}^{3}, \alpha-1=\beta_{2}^{3}$ and $\beta_{1}, \beta_{2}$ are integers. The solutions of this equation do not provide desired $\alpha$.

If $n \equiv 6,24(30)$, then $h_{n}=C$ iff

$$
\alpha(2 \alpha-1)^{2}\left(-\alpha^{2}+3 \alpha-1\right)^{2}=C .
$$

This equation leads to classical equation

$$
\left(-\beta_{2}\right)^{3}+2 \beta_{1}^{3}=1
$$

where $\alpha=\beta_{1}^{3}, 2 \alpha-1=\beta_{2}^{3}$ and $\beta_{1}, \beta_{2}$ are integers. The only solution of this equation is $\left(\beta_{1}, \beta_{2}\right)=(1,1)$ and this solution does not provide desired $\alpha$.

5.8. The Case $N=12$. We determine square terms in the following theorem.

Theorem 5.23. Let $\left(h_{n}\right)$ be an elliptic divisibility sequence for which the twelfth term is zero.

i. If $n \equiv 1,23$ (24), then $h_{n}=\square$.

ii. If $n \equiv 5,19(24)$, then $h_{n}=\square$ iff $3 \alpha^{2}-3 \alpha+1=\square$.

Proof. The case $(i)$ can be proved in the same way as in Theorem 5.1. If $n \equiv$ 5,19 (24), then $h_{n}=\square$ iff

$$
3 \alpha^{2}-3 \alpha+1=
$$

by (3.9). It follows that

$$
\beta^{2}-3(2 \alpha-1)^{2}=1
$$


or

$$
\beta^{2}+3(2 \alpha-1)^{2}=-1
$$

where $\beta$ is an integer. The last equation is impossible modulo 3 , and the first leads to Pell equation. If we rewrite equation (5.3) as

$$
(\beta+(2 \alpha-1) \sqrt{3})(\beta-(2 \alpha-1) \sqrt{3})=1
$$

we see that the solutions of this equation are $(2,1),(7,4),(26,15), \ldots$ and parameters $\alpha$ corresponding to these solutions are $1, \frac{5}{2}, 8, \ldots$. It can easily be seen that the only odd solutions of this Pell equation gives the desired solution of $\alpha$, i.e., for such an $\alpha, 3 \alpha^{2}-3 \alpha+1$ is a square and their number is infinite.

Theorem 5.24. Let $\left(h_{n}\right)$ be an elliptic divisibility sequence for which the twelfth term is zero. If $n \equiv 2,3,4,6,7,8,9,10,11,13,14,15,16,17,18,20,21,22$ (24) then $h_{n} \neq \square$ for all $\alpha \in \mathbb{Z} \backslash\{0,1\}$.

Proof. If $n \equiv 2,3,10,14,21,22(24)$, then $h_{n}=\square$ iff

$$
\alpha(2 \alpha-1)\left(2 \alpha-2 \alpha^{2}-1\right)\left(3 \alpha^{2}-3 \alpha+1\right)=
$$

by (3.9). This equation leads to

$$
(3 \alpha)^{3}-3(3 \alpha)^{2}+3(3 \alpha)=\beta^{2}
$$

or

$$
\alpha(2 \alpha-1)=-\beta^{2}
$$

where $\beta$ is an integer. From the first equation we have an elliptic curve with rank 0 and torsion points on this curve do not provide desired $\alpha$. The last equation is impossible since $\alpha$ is an integer.

If $n \equiv 4,8,16,20(24)$, then $h_{n}=\square$ iff

$$
(\alpha-1)\left(2 \alpha-2 \alpha^{2}-1\right)=
$$

by (3.9). This equation leads to

$$
(-2 \alpha)^{3}+4(-2 \alpha)^{2}+6(-2 \alpha)+4=\beta^{2}
$$

or

$$
(2 \alpha)^{3}-4(2 \alpha)^{2}+6(2 \alpha)-4=\beta^{2}
$$

where $\beta$ is an integer. From the last equation we have an elliptic curve with rank 0 and torsion points on this curve do not provide desired $\alpha$. From the first equation we have an elliptic curve with rank 1 and applying the Elliptic Logarithm Method we see that the only solutions to this equation are $\alpha=0,1$ and -3 . So we have $\alpha=-3$.

If $n \equiv 6,18(24)$, then $h_{n}=\square$ iff

$$
\alpha\left(3 \alpha^{2}-3 \alpha+1\right)=\square
$$

if $n \equiv 11,13(24)$, then $h_{n}=\square$ iff

$$
\alpha(\alpha-1)\left(3 \alpha^{2}-3 \alpha+1\right)=\square .
$$

by (3.9), or equivalently $\alpha\left(3 \alpha^{2}-3 \alpha+1\right)=\square$. This equation leads to

$$
(3 \alpha)^{3}-3(3 \alpha)^{2}+3(3 \alpha)=\beta^{2}
$$


Or

$$
(-3 \alpha)^{3}+3(-3 \alpha)^{2}+3(-3 \alpha)=\beta^{2} .
$$

where $\beta$ is an integer. From these equations we have elliptic curves with rank 0 and torsion points on these curves do not provide desired $\alpha$.

If $n \equiv 7,17(24)$, then $h_{n}=\square$ iff

$$
\alpha(\alpha-1)=\square .
$$

But this is impossible by proof of Theorem 5.10.

If $n \equiv 9,15(24)$, then $h_{n}=\square$ iff

$$
(\alpha-1)(2 \alpha-1)\left(2 \alpha-2 \alpha^{2}-1\right)=\square .
$$

by (3.9). This equation leads to

$$
(4 \alpha)^{3}-6(4 \alpha)^{2}+16(4 \alpha)-16=\beta^{2}
$$

or

$$
(-4 \alpha)^{3}+6(-4 \alpha)^{2}+16(-4 \alpha)+16=\beta^{2}
$$

where $\beta$ is an integer. From these equations we have elliptic curves with rank 0 and torsion points on this curves do not provide desired $\alpha$.

An easy calculation as in Theorems 5.1 and 5.2 gives the following theorem.

Theorem 5.25. Let $\left(h_{n}\right)$ be an elliptic divisibility sequence for which the twelfth term is zero.

i. If $n \equiv 1,35$ (36), then $h_{n}=C$.

ii. If $n \equiv 3,9,15,21,27,33(36)$, then $h_{n}=C$ iff $\alpha-1=C$.

Theorem 5.26. Let $\left(h_{n}\right)$ be an elliptic divisibility sequence for which the twelfth term is zero. If $n \equiv 2,4,5,6,7,8,10,11,13,14,16,17,18,19,20,22,23,25$, 26, 28, 29, 30, 31, 32, 34 (30) then $h_{n} \neq C$ for all $\alpha \in \mathbb{Z} \backslash\{0,1\}$.

Proof. If $n \equiv 2,34$ (36), then $h_{n}=C$ iff

$$
\alpha(2 \alpha-1)(\alpha-1)^{2}\left(2 \alpha-2 \alpha^{2}-1\right)\left(3 \alpha^{2}-3 \alpha+1\right)=C,
$$

if $n \equiv 8,28(36)$, then $h_{n}=C$ iff

$$
\alpha(\alpha-1)^{2}(2 \alpha-1)^{2}=C,
$$

if $n \equiv 11,25(36)$, then $h_{n}=C$ iff

$$
\alpha(\alpha-1)(2 \alpha-1)^{2}\left(2 \alpha-2 \alpha^{2}-1\right)^{2}=C,
$$

if $n \equiv 13,23(36)$, then $h_{n}=C$ iff

$$
\alpha^{2}(\alpha-1)^{2}(2 \alpha-1)\left(2 \alpha-2 \alpha^{2}-1\right)^{2}=C,
$$

if $n \equiv 17,19(36)$, then $h_{n}=C$ iff

$$
\alpha^{2}(\alpha-1)=C
$$

by (3.9). These equations lead to trivial equation

$$
\beta_{1}^{3}-\beta_{2}^{3}=1
$$

where $\alpha=\beta_{1}^{3}, \alpha-1=\beta_{2}^{3}$ and $\beta_{1}, \beta_{2}$ are integers. The solutions of this equation do not provide desired $\alpha$. 
If $n \equiv 4,32(36)$, then $h_{n}=C$ iff

$$
(\alpha-1)\left(2 \alpha-2 \alpha^{2}-1\right)^{2}=C,
$$

if $n \equiv 14,22(36)$, then $h_{n}=C$ iff

$$
\alpha^{2}\left(2 \alpha-2 \alpha^{2}-1\right)^{2}\left(3 \alpha^{2}-3 \alpha+1\right)=C .
$$

by (3.9). These equations lead to

$$
\beta^{3}-4=(4 \alpha-2)^{2}
$$

where $\beta$ is an integer. From this equation we have an elliptic curve with rank 1 and applying the Elliptic Logarithm Method we see that the integral points on this curve do not provide desired $\alpha$.

If $n \equiv 5,31$ (36), then $h_{n}=C$ iff

$$
\alpha(2 \alpha-1)\left(2 \alpha-2 \alpha^{2}-1\right)^{2}=C,
$$

if $n \equiv 16,20(36)$, then $h_{n}=C$ iff

$$
\alpha^{2}(2 \alpha-1)\left(2 \alpha-2 \alpha^{2}-1\right)=C,
$$

by (3.9). These equations lead to classical equation

$$
\left(-\beta_{2}\right)^{3}+2 \beta_{1}^{3}=1
$$

where $\alpha=\beta_{1}^{3}, 2 \alpha-1=\beta_{2}^{3}$ and $\beta_{1}, \beta_{2}$ are integers. The only solution of this equation is $\left(\beta_{1}, \beta_{2}\right)=(1,1)$ and this solution does not provide desired $\alpha$.

If $n \equiv 6,18,30(36)$, then $h_{n}=C$ iff

$$
(\alpha-1)^{2}(2 \alpha-1)^{2}\left(3 \alpha^{2}-3 \alpha+1\right)=C,
$$

if $n \equiv 7,29$ (36), then $h_{n}=C$ iff

$$
(\alpha-1)^{2}(2 \alpha-1)^{2}\left(2 \alpha-2 \alpha^{2}-1\right)=C,
$$

if $n \equiv 10,26(36)$, then $h_{n}=C$ iff

$$
(\alpha-1)(2 \alpha-1)^{2}\left(3 \alpha^{2}-3 \alpha+1\right)=C,
$$

by (3.9). These equations lead to classical equation

$$
\beta_{2}{ }^{3}+2\left(-\beta_{1}\right)^{3}=1
$$

where $\alpha-1=\beta_{1}^{3}, 2 \alpha-1=\beta_{2}^{3}$ and $\beta_{1}, \beta_{2}$ are integers. The solution of this equation is $\left(\beta_{1}, \beta_{2}\right)=(-1,-1)$ and this solution does not provide desired $\alpha$.

Acknowledgements. The author would like to thank Joseph H. Silverman for looking at the initial draft and making suggestions for the first parts of the paper. Also I am grateful to Nikos Tzanakis for his helpful suggestions for improving the paper. 


\section{REFERENCES}

[1] Bosma, W., Cannon, J., Playoust, C., The Magma Algebra System I. The user language, J. Symbolic Comput. 24 (3-4), 235-265 (1997)

[2] Bremner, A., Tzanakis, N., Lucas sequences whose 12th or 9th term is a square, J. Number Theory 107, 215-227 (2004).

[3] Bremner, A., Tzanakis, N., On squares in Lucas sequences, J. Number Theory 24, 511-520 (2007).

[4] Einsiedler, M., Everest, G., Ward, T., Primes in elliptic divisibility sequences, LMS J. Comput. Math. 4, 1-13, electronic (2001).

[5] Everest, G., van der Poorten, A., Shparlinski, I., Ward, T., Recurrence sequences, Mathematical Surveys and Monographs 104, AMS, Providence, RI, 2003.

[6] Garcia-Selfa, I., Olalla, M. A., Tornero, J. M., Computing the rational torsion of an elliptic curve using Tate normal form, Journal of Number Theory, 96, (2002), 76-88.

[7] Gebel, J., Pethő, A., Zimmer, H. G., Computing integral points on elliptic curves, Acta Arithmetica, 68, 171-192 (1994).

[8] Gezer, B., Bizim, O., Squares in Elliptic Divisibility Sequences, Acta Arithmetica, 2, 144, 125-134, (2010).

[9] Gezer, B., Bizim, O., Cubes in Elliptic divisibility sequences, Mathematical Reports, accepted for publication.

[10] Husemöller, D., Elliptic Curves, Springer Verlag, New York, 1987.

[11] Kubert, D. S., Universal bounds on the torsion of elliptic curves. Proc. London Math. Soc., 33 (3) (1976), 193-237.

[12] Mazur, B., Modular curves and the Eisenstein ideal, IHES Publ. Math. 47 (1977), 33-186.

[13] http://magma.maths.usyd.edu.au/calc/

[14] Mordell, L. J., Diophantine Equations, Pure and Applied Mathematics 30, Academic Press, London and New York 1970.

[15] http://pari.maths.u-bordeaux.fr/

[16] Pethö, A., Full cubes in the Fibonacci sequence, Publ. Math. Debrecen 30, 117-127 (1983).

[17] Ribenboim, P., Pell numbers, squares and cubes, Publ. Math. Debrecen 54, 131-152 (1999).

[18] Ribenboim, P., McDaniel, W., The square terms in Lucas sequences, J. Number Theory 58, 104-123 (1996).

[19] Ribenboim, P., McDaniel, W., Squares in Lucas sequences having an even first parameter, Colloquium Mathematicum 78, 29-34 (1998).

[20] Shipsey, R., Elliptic divisibility sequences, Ph. D. thesis, Goldsmith's (University of London), 2000.

[21] Silverman, J. H., The Arithmetic of Elliptic Curves 2nd Edition, Graduate Texts in Mathematics 106, Springer Dordrecht Heidelberg London New York 2009.

[22] Silverman, J. H., Tate, J., Rational Points on Elliptic Curves, Undergraduate Texts in Mathematics, Springer, 1992.

[23] Stroeker, R. J., Tzanakis N., Solving elliptic diophantine equations by estimating linear forms in elliptic logarithms, Acta Arithmetica, 67, 177-196 (1994).

[24] Swart, C. S., Elliptic curves and related sequences, Ph. D. thesis, Royal Holloway (University of London), 2003.

[25] Ward, M., The law of repetition of primes in an elliptic divisibility sequences, Duke Math. J. 15 (1948), 941-946.

[26] Ward, M., Memoir on elliptic divisibility sequences, Amer. J. Math. 70 (1948), 31-74.

Uludag University, Faculty of Science, Department of Mathematics, Görükle, 16059, BURSA-TURKEY

E-mail address: betulgezer@uludag.edu.tr 